\title{
ANTI-ANGIOGENIC THERAPY IN CANCER TREATMENT AS AN OPTIMAL CONTROL PROBLEM *
}

\author{
URSZULA LEDZEWICZ ${ }^{\dagger}$ AND HEINZ SCHÄTTLER ${ }^{\ddagger}$
}

\begin{abstract}
Anti-angiogenic therapy is a novel treatment approach in cancer therapy that aims at preventing a tumor from developing its own blood supply system that it needs for growth. In this paper a mathematical model for anti-angiogenic treatments based on a biologically validated model by Hahnfeldt, Panigrahy, Folkman and Hlatky is analyzed as an optimal control problem and a full solution of the problem is given. Geometric methods from optimal control theory are utilized to arrive at the solution.
\end{abstract}

Key words. optimal control, geometric methods, cancer treatment, anti-angiogenic therapy

AMS subject classifications. 49K15, 92C50, 37N25

1. Introduction. The most important limiting factor for the success of cancer chemotherapy treatments lies in both intrinsic and acquired drug resistance. Malignant cancer cell populations are highly heterogeneous - the number of genetic errors present within one cancer cell can lie in the thousands [16] - and fast duplications combined with genetic instabilities provide just one of several mechanisms which allow for quickly developing acquired resistance to anti-cancer drugs. In addition, intrinsic resistance (i.e. the specific drug's activation mechanism simply doesn't work) makes some cancer cells not susceptible to many cytotoxic agents. " ... the truly surprising thing is that some malignancies can be cured even with current approaches." [8, pp. 65]. Several mechanisms to circumvent the problem of drug resistance have been tried, but so far without success and currently no medical solution to the problem exists. In fact, it is acquired or intrinsic drug resistance which eventually makes most chemotherapy fail. At the same time, similar phenomena do not take place for the healthy proliferating cells. For example, regretfully bone marrow does not develop drug resistance to the killing agent [10].

As of today, the search for therapy approaches that would avoid drug resistance still is of tantamount importance in medicine. Two such approaches that are currently being pursued in their experimental stages are immunotherapy and anti-angiogenic treatments. While immunotherapy tries to coax the body's immune system to take action against the cancerous growth, tumor anti-angiogenesis aims at depriving a tumor from developing the necessary blood cells and capillaries that it needs for further growth. Since the treatment does not target cancer cells, but normal cells, no occurrence of drug resistance has been reported in lab studies. (These treatments, however, are only in the stage of experimental studies and initial clinical trials.) For this reason tumor anti-angiogenesis has been called a therapy resistant to resistance which provides a new hope in treatment of tumor type cancers [10].

There exist several mathematical models for the evolution of tumor anti-angiogenesis as a dynamical system with the one formulated by Hahnfeldt, Panigrahy, Folkman

*This material is based upon research supported by the National Science Foundation under collaborative research grants DMS 0405827/0405848. U. Ledzewicz's research is also partially supported by SIUE 2006 Summer Research Fellowship.

${ }^{\dagger}$ Dept. of Mathematics and Statistics, Southern Illinois University at Edwardsville, Edwardsville, Illinois, 62026-1653, (uledzew@siue.edu)

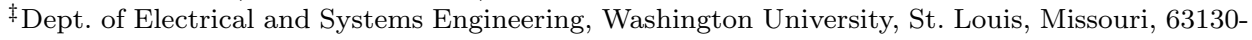
4899, (hms@wustl.edu) 
and Hlatky in [9] probably being the most prominent one. This model was biologically validated in lab experiments and became the basis for several modifications and simplifications $[5,6]$ undertaken in an effort to both better understand the dynamical properties of the underlying mechanisms and to make the mathematical model easier and more tractable for analysis. For example, a dynamical systems analysis of the model by Hahnfeldt et al. and of several modifications (with more general growth models for the growth of cancer cells and slightly different dynamics for the evolution related to endothelial cells) is given in the paper by d'Onofrio and Gandolfi [5]; Ergun, Camphausen and Wein [6] consider an optimal control problem for the scheduling of anti-angiogenic inhibitors both as monotherapy and in combination with radiotherapy. While these models are variations of the specific dynamics proposed by Hahnfeldt et al. in [9], in the papers by Agur, Arakelyan, Daugulis and Ginosar [1] and Forys, Kheifetz and Kogan [7] more generally dynamical properties of models for angiogenesis are investigated under minimal assumptions on the form of the growth functions describing the dynamics.

In this paper we consider the original mathematical model for tumor anti-angiogenesis formulated and validated by Hahnfeldt, Panigrahy, Folkman and Hlatky in [9] and analyze it as an optimal control problem. Using geometric methods of optimal control theory, for this model we compute how to schedule a given amount of angiogenic inhibitors to achieve the maximum reduction in tumor volume possible. The key feature of the solution is an optimal singular arc whose geometric analysis forms the core of the mathematical argument. Optimal controls then are concatenations of bang controls (constant controls that either give a full or no dose of inhibitors) and the optimal singular control (a specific smooth control that administers the inhibitors using a time varying feedback schedule at less than a maximum rate). The most general structure of optimal controls possible is a concatenation of the form "0asa0" where $\mathbf{a}$ and $\mathbf{0}$ denote trajectories with full, respectively no anti-angiogenic therapy and $\mathbf{s}$ stands for a segment along the singular arc. However, depending on the initial condition not all of these pieces are present. Our theoretical analysis reduces the structure of optimal controls to at most this structure, but for some initial conditions still allows for a one-parameter family of extremals of this form. Then, given any initial condition, the optimal solution is easily computed numerically based on our analysis. The most typical and medically most relevant scenarios are optimal protocols that take the simple form "a0" when all inhibitors are administered at the beginning or "as0" when the dosage is adjusted as the singular arc is reached and then all available inhibitors are being used up along the singular arc. If the optimal policy along the singular arc comes close to a point where the singular control saturates at the upper value $a$, then optimal trajectories actually leave the singular arc prior to saturation (and this is consistent with the behavior of optimal controls near saturation points, see, for example [17] or [2]) and are of the type "asa0". The full structure "0asa0" only arises for initial condition that are not significant for the underlying problem.

A preliminary announcement of some partial results presented in this paper has been given without proofs in [14]. Here the analysis is completed and proofs are included.

2. Medical Background and Mathematical Model [9]. A growing tumor, after it reaches just a few millimeters in diameter, no longer can rely on blood vessels of the host for its supply of nutrients, but it needs to develop its own vessels and capillaries for blood supply. In this process, called angiogenesis, there is a reciprocal signaling between endothelial cells and tumor cells. Tumor cells produce vascular 
endothelial growth factor (VEGF) to stimulate endothelial cell growth; endothelial cells in turn provide the lining for the newly forming blood vessels that supply nutrients to the tumor and thus sustain tumor growth. But endothelial cells also have receptors which make them sensitive to inhibitors of inducers of angiogenesis like, for example, endostatin, and pharmacologic therapies typically target the growth factor VEGF trying to impede the development of new blood vessels and capillaries. Overall, angiogenesis can be viewed as a complex balance of stimulatory and inhibitory mechanisms regulated through microenvironmental factors.

In the model developed by Hahnfeldt, Panigrahy, Folkman and Hlatky in [9] these effects are summarized in a two-dimensional dynamical system with the primary tumor volume, $p$, and the carrying capacity of the vasculature, $q$, as variables. The latter is defined as the "maximal tumor volume potentially sustainable by the network" [9] and is implicitely assumed proportional to the number of endothelial cells. Thus the set $\mathcal{D}_{0}=\left\{(p, q) \in \mathbb{R}_{+}^{2}: p=q\right\}$ corresponds to points where the vasculature is adequate to support the tumor, while $\mathcal{D}_{-}=\{(p, q) \in \mathcal{D}: p<q\}$ corresponds to growing tumors and $\mathcal{D}_{+}=\{(p, q) \in \mathcal{D}: p>q\}$ to shrinking tumors. A growth function describes the size of the tumor dependent on the carrying capacity $q$ and is chosen as Gompertzian in the original model. Other models are equally realistic and are considered, for instance, in [5] or [7], but here we stay with the original choice. Thus the rate of change in the primary tumor volume is modelled as

$$
\dot{p}=-\xi p \ln \left(\frac{p}{q}\right)
$$

where $\xi$ denotes a tumor growth parameter. The overall dynamics for the carrying capacity is a balance between stimulation and inhibition and its basic structure is of the form

$$
\dot{q}=-\mu q+S(p, q)-I(p, q)-G u q
$$

where $\mu q$ describes the loss of endothelial cells due to natural causes (death etc.), $I$ and $S$ denote endogenous inhibition and stimulation terms, respectively, and Guq represents a loss due to additional outside inhibition. The variable $u$ represents the control in the system and corresponds to the angiogenic dose rate while $G$ is a constant that represents the anti-angiogenic killing parameter. Generally $\mu$ is small and often this term is negligible compared to the other factors and thus in the literature often $\mu$ is set to 0 in this equation.

In [9] a spatial analysis of the underlying consumption-diffusion model was carried out that led to the following two principal conclusions:

1. The inhibitor will impact endothelial cells in a way that grows like volume of cancer cells to the power $\frac{2}{3}$.

The exponent $\frac{2}{3}$ arises since inhibitors need to be released through the surface of the tumor. Thus in [9] the inhibitor term is taken in the form

$$
I(p, q)=d p^{\frac{2}{3}} q
$$

with $d$ a constant, the 'death' rate. The second implication of the analysis in [9] is that:

2. The inhibitor term will tend to grow at a rate of $q^{\alpha} p^{\beta}$ faster than the stimulator term where $\alpha+\beta=\frac{2}{3}$. 
However, the choice of $\alpha$ and $\beta$ is not imperative in their analysis and in fact is one of the main sources for the various other models also considered in the literature $[5,6]$. In their original work [9] Hahnfeldt et al. select $\alpha=1$ and $\beta=-\frac{1}{3}$ resulting in the simple stimulation term

$$
S(p, q)=b p
$$

with $b$ a constant, the 'birth' rate. However, other choices are possible and, for example, choosing $\alpha=0$ and $\beta=\frac{2}{3}$ results in the equally simple form $S(p, q)=b q$ chosen in [5]. In that paper the dynamics for both models is analyzed and it is shown for the uncontrolled system that there exists a unique globally asymptotically stable equilibrium (which, of course, is not viable biologically). Adding a control term, this equilibrium can be shifted to lower values, or, depending on the parameter values, even eliminated altogether. In the latter case all trajectories converge to the origin in infinite time. This, in principle, would be the desired situation.

The problem then becomes how to administer a given amount of inhibitors to achieve the "best possible" effect. In this paper we use the dynamics of the original model from [9] and formulate this aim as the following optimal control problem:

[HPFH] For a free terminal time $T$, minimize the value $p(T)$ subject to the dynamics

$$
\begin{array}{ll}
\dot{p}=-\xi p \ln \left(\frac{p}{q}\right), & p(0)=p_{0}, \\
\dot{q}=b p-\left(\mu+d p^{\frac{2}{3}}\right) q-G u q, & q(0)=q_{0}, \\
\dot{y}=u, & y(0)=0,
\end{array}
$$

over all measurable functions $u:[0, T] \rightarrow[0, a]$ for which the corresponding trajectory satisfies $y(T) \leq A$.

As it is customary in optimal control formulations, we adjoin the constraint as third variable. Later on, for all our numerical illustrations we use the following parameter values which are taken from [9]: The variables $p$ and $q$ are volumes measured in $\mathrm{mm}^{3} ; \xi=\frac{0.192}{\ln 10}=0.084$ per day (adjusted to the natural logarithm), $b=5.85$ per day, $d=0.00873$ per $\mathrm{mm}^{2}$ per day, $G=0.15 \mathrm{~kg}$ per $\mathrm{mg}$ of dose per day, and for illustrative purposes we chose a small positive value for $\mu, \mu=0.02$ per day. But we want to emphasize already that our mathematical analysis and conclusions are valid independently of the specific parameter values and lead to robust implications about the structure of optimal controls for this model.

3. The Dynamical Systems for Constant Controls. For the analysis of the optimal control problem it is of benefit to fully understand the dynamic properties of the systems for a constant control $u \equiv v$ with $v$ some value in the control set $[0, a]$. Our statements in this section are only minor extensions of the analysis given in the paper by d'Onofrio and Gandolfi [5] and we refer the reader to this paper for the proofs about our claims of stability properties of the equilibria. All statements are for the natural domain $\mathbb{R}_{+}^{2}=\{(p, q): p>0, q>0\}$ of the system. The following fact about the dynamical behavior of the system is an easy corollary of the results proven in $[5]$ :

Proposition 3.1. For any admissible control $u$ and arbitrary positive initial conditions $p_{0}$ and $q_{0}$, the corresponding solution $(p, q)$ exists for all times $t \geq 0$ and both $p$ and $q$ remain positive.

Assuming $b>\mu$, the uncontrolled system $(u=0)$ has a unique globally asymptotically stable equilibrium point at $(\bar{p}, \bar{q})$ given by $\bar{p}=\bar{q}=\left(\frac{b-\mu}{d}\right)^{\frac{3}{2}}$. This value naturally 
is far too high to be acceptable and the medically relevant region is contained in the domain

$$
\mathcal{D}=\{(p, q): 0<p \leq \bar{p}, 0<q \leq \bar{q}\}
$$

In order to exclude irrelevant discussions about the structure of optimal controls in regions where the model does not represent the underlying medical problem to begin with, we henceforth restrict our discussions to this square domain $\mathcal{D}$.

Proposition 3.2. $\mathcal{D}$ is positively invariant for the flow of the control system, i.e. if $\left(p_{0}, q_{0}\right) \in \mathcal{D}$, then for any admissible control $u$ defined over the interval $[0, \infty)$ the solution $(p(\cdot), q(\cdot))$ to the corresponding dynamics with initial condition $(p(0), q(0))=$ $\left(p_{0}, q_{0}\right)$ exists for all times $t \geq 0$ and lies in $\mathcal{D},(p(t), q(t)) \in \mathcal{D}$.

Proof. The positive invariance of the region $\mathcal{P}=\{(p, q): 0<p, 0<q\}$ for any admissible control $u$ directly follows from Proposition 3.1. The dynamics is clearly pointing into $\mathcal{D}$ on the boundary segment $\{(p, q) \in \mathcal{D}: p=\bar{p}, 0<q<\bar{q}\}$ since for $p>q$ we always have $\dot{p}<0$. For a constant control $v$ the isoclines for $\dot{q}=0$ are given by

$$
q=\Xi_{v}(p)=\frac{b p}{\mu+G v+d p^{\frac{2}{3}}} .
$$

The functions $\Xi_{v}$ are strictly increasing, $\Xi_{v}(0)=0$, and at $\bar{p}$ take the value

$$
\Xi_{v}(\bar{p})=\frac{b}{b+G v} \bar{p} .
$$

In particular, the smallest value is given for $v=a$ and $\Xi_{0}(\bar{p})=\bar{p}$. It thus follows that on the boundary segment $\{(p, q) \in \mathcal{D}: 0<p<\bar{p}, q=\bar{q}\}$ we have $\dot{q}<0$ for all controls. The point $(\bar{p}, \bar{q})$ is the equilibrium point for $u=0$ and the dynamics points into $\mathcal{D}$ for $u=a$ at this point. Thus, regardless of the control value $v$, trajectories can never leave the region $\mathcal{D}$.

By increasing the value $v$ of the control, the equilibrium can be shifted towards the origin along the diagonal and finally be eliminated altogether. As a function of $v$ the equilibrium is the unique fixed point of the equation $p=\Xi_{v}(p)$ in $\{p>0\}$ and is given by

$$
\bar{p}(v)=\bar{q}(v)=\left(\frac{b-\mu-G v}{d}\right)^{\frac{3}{2}}
$$

provided $b-\mu>G v$ and this equilibrium $(\bar{p}(v), \bar{q}(v))$ still is globally asymptotically stable. As $b-\mu \leq G v$, the system no longer has an equilibrium point and now all trajectories converge to the origin as $t \rightarrow \infty$ [5]. Thus, theoretically eradication of the tumor were possible in this case under the unrealistic scenario of constant treatment with unlimited supply of inhibitors. Since this is the most desirable situation, for our analysis of the optimal control problem we also assume that

$$
\text { (A) } \quad \mathbf{G a}>\mathbf{b}-\mu>\mathbf{0} \text {. }
$$

Fig. 3.1 shows the phase portraits of the uncontrolled system on the left and for $u \equiv a$ on the right. In our figures we prefer to have the tumor volume as the vertical axis since this better visualizes the tumor reduction respectively increase. For comparison the diagonal is included in these figures as a dash-dotted line. It is not difficult to 



FIG. 3.1. Phase portraits for $u=0$ and $u=a=75$

extend all results of this paper to the case when the dynamics for $u \equiv a$ still has a positive equilibrium, but this will not be pursued here for reasons of space.

However, the domain $\mathcal{D}$ still contains initial conditions that give rise to degenerate cases that we want to exclude. Recall that $\mathcal{D}_{+}=\{(p, q) \in \mathcal{D}: p>q\}, \mathcal{D}_{0}=\{(p, q) \in$ $\mathcal{D}: p=q\}$ and $\mathcal{D}_{-}=\{(p, q) \in \mathcal{D}: p<q\}$. Both the trajectories for the constant controls $u=0$ and $u=a$ cross the diagonal portion $\mathcal{D}_{0}$ transversally: for $u=0$ trajectories cross from $\mathcal{D}_{+}$into $\mathcal{D}_{-}$, while they cross in opposite direction from $\mathcal{D}_{-}$ into $\mathcal{D}_{+}$for $u=a$. Also, trajectories for $u=0$ approach the stable equilibrium $(\bar{p}, \bar{q})$ from within the region $\mathcal{D}_{-}$, while trajectories for $u=a$ converge to the origin as $t \rightarrow \infty$ in the region $\mathcal{D}_{+}$. It follows from the dynamics for $p,(2.5)$, that the $p$ value of trajectories is always decreasing in $\mathcal{D}_{+}$and always increasing in $\mathcal{D}_{-}$. As a result, for some initial conditions $\left(p_{0}, q_{0}, y_{0}\right)$ with $\left(p_{0}, q_{0}\right) \in \mathcal{D}_{-}$, it is possible that the (mathematically) optimal time $T$ is $T=0$. This situation arises when the amount of available inhibitors simply is not sufficient to reach a point in the region $\mathcal{D}_{+}$that would have a lower $p$-value than $p_{0}$. In such a case it is not possible to decrease the tumor volume with the available amount of inhibitors. It is only possible to slow down the tumor's growth. Indeed, it is correct that the best way of doing this is to give the full dose $u=a$ until all inhibitors run out - this follows from the structure of optimal controls to be shown later - but this is not the mathematically "optimal" solution for problem [HPFH]. This one is simply to do nothing and take $T=0$. Since this introduces a number of degeneracies into the analysis, we make the following definition:

Definition 3.3. We say an initial condition $\left(p_{0}, q_{0}\right) \in \mathcal{D}_{-}$is ill-posed if for any admissible control it is not possible to reach a point $(p, q)$ with $p<p_{0}$. In this case the optimal solution for the problem [HPFH] is given by $T=0$. Otherwise $\left(p_{0}, q_{0}\right)$ is well-posed and the optimal time $T$ will be positive.

It is clear that all initial conditions with $\left(p_{0}, q_{0}\right) \in \mathcal{D}_{+} \cup \mathcal{D}_{0}$ are well-posed (since $p$ decreases in $\mathcal{D}_{+}$and trajectories with $u=a$ enter $\mathcal{D}_{+}$from $\mathcal{D}_{0}$ ) and it is easily decided whether an initial condition $\left(p_{0}, q_{0}\right) \in \mathcal{D}_{-}$is ill-posed. For our analysis of optimal controls, however, we only consider well-posed initial conditions.

4. The Maximum Principle and Preliminary Analysis of Optimal Controls. It follows from classical results that there exists an optimal solution to our problem [4]. First-order necessary conditions for optimality of a control $u$ are given 
by the Pontryagin Maximum Principle [18, 3, 4]: If $u_{*}$ is an optimal control defined over an interval $[0, T]$ with corresponding trajectory $\left(p_{*}, q_{*}, y_{*}\right)^{T}$, then there exist a constant $\lambda_{0} \geq 0$ and an absolutely continuous co-vector, $\lambda:[0, T] \rightarrow\left(\mathbb{R}^{3}\right)^{*}$, (which we write as row-vector) such that (a) $\left(\lambda_{0}, \lambda(t)\right) \neq(0,0)$ for all $t \in[0, T]$, (b) the adjoint equations hold with transversality conditions,

$$
\begin{array}{ll}
\dot{\lambda}_{1}=\xi \lambda_{1}\left(\ln \left(\frac{p_{*}(t)}{q_{*}(t)}\right)+1\right)+\lambda_{2}\left(\frac{2}{3} d \frac{q_{*}(t)}{p_{*}^{\frac{1}{3}}(t)}-b\right), & \lambda_{1}(T)=\lambda_{0}, \\
\dot{\lambda}_{2}=-\xi \lambda_{1} \frac{p_{*}(t)}{q_{*}(t)}+\lambda_{2}\left(\mu+d p_{*}^{\frac{2}{3}}(t)+G u\right), & \lambda_{2}(T)=0, \\
\dot{\lambda}_{3}=0, & \lambda_{3}(T)=\left\{\begin{array}{cc}
0 & \text { if } y(T)<A \\
\text { free } & \text { if } y(T)=A
\end{array},\right.
\end{array}
$$

and (c) the optimal control $u_{*}$ minimizes the Hamiltonian $H$,

$$
H=-\lambda_{1} \xi p \ln \left(\frac{p}{q}\right)+\lambda_{2}\left(b p-\left(\mu+d p^{\frac{2}{3}}\right) q-G u q\right)+\lambda_{3} u
$$

along $\left(\lambda(t), p_{*}(t), q_{*}(t)\right)$ over the control set $[0, a]$ with minimum value given by 0 .

We call a pair $((p, q, y), u)$ consisting of an admissible control $u$ with corresponding trajectory $(p, q, y)$ an extremal (pair) if there exist multipliers $\left(\lambda_{0}, \lambda\right)$ such that the conditions of the Maximum Principle are satisfied and the triple $\left((p, q, y), u,\left(\lambda_{0}, \lambda\right)\right)$ is an extremal lift (to the cotangent bundle). Extremals with $\lambda_{0}=0$ are called abnormal while those with a positive multiplier $\lambda_{0}$ are called normal. In this case it is possible to normalize $\lambda_{0}=1$. The following Lemmas summarize some elementary properties of optimal controls and extremals for well-posed initial conditions.

LEMMA 4.1. If $u_{*}$ is an optimal control with corresponding trajectory $\left(p_{*}, q_{*}, y_{*}\right)^{T}$, then at the final time $p_{*}(T)=q_{*}(T)$ and $y_{*}(T)=A$, i.e. all available inhibitors have been used up.

Proof. Since the $p$-dynamics is Gompertzian, (2.5), the cancer volume is growing for $p<q$ and is shrinking for $p>q$. This implies that optimal trajectories can only terminate at times where $p_{*}(T)=q_{*}(T)$. For, if $p_{*}(T)<q_{*}(T)$, then it would simply have been better to stop earlier since $p$ was increasing over some interval $(T-\varepsilon, T]$. (Recall that we are assuming that the initial condition is well-posed so that the optimal final time $T$ is positive.) On the other hand, if $p_{*}(T)>q_{*}(T)$, then we can always add another small interval $(T, T+\varepsilon]$ with the control $u=0$ without violating any of the constraints and $p$ will decrease along this interval if $\varepsilon$ is small enough. Thus at the final time necessarily $p_{*}(T)=q_{*}(T)$. If now $y(T)<A$, then we can still add a small piece of a trajectory for $u=a$ over some interval $[0, \varepsilon]$. Since $\dot{q}<0$ on the diagonal $\mathcal{D}_{0}$ the corresponding trajectory lies in $\mathcal{D}_{+}$and thus the value of $p$ is decreasing along this trajectory contradicting the optimality of $T$.

LEMMA 4.2. Extremals are normal. The multipliers $\lambda_{1}$ and $\lambda_{2}$ cannot vanish simultaneously; $\lambda_{2}$ has only simple zeroes. The multiplier $\lambda_{3}$ is constant and nonnegative.

Proof. The multipliers $\lambda_{1}$ and $\lambda_{2}$ satisfy the homogeneous linear system (4.1) and (4.2) and thus they vanish identically if they vanish at some time $t$. If $\lambda_{0}=0$, then the nontriviality of $\left(\lambda_{0}, \lambda(t)\right)$ implies that the multiplier $\lambda_{3}$, which is constant, is not zero. The condition $H \equiv 0$ on the Hamiltonian therefore gives $u \equiv 0$, i.e. the initial 
condition is ill-posed. Thus, without loss of generality we may assume that $\lambda_{0}=1$ and hence $\lambda_{1}$ and $\lambda_{2}$ cannot vanish simultaneously. In particular, whenever $\lambda_{2}(t)=0$, then $\dot{\lambda}_{2}(t) \neq 0$ and thus $\lambda_{2}$ has only simple zeroes.

For the final time $T$ it follows from $p_{*}(T)=q_{*}(T)$, the transversality condition $\lambda_{2}(T)=0$, and the condition $H(T) \equiv 0$ that $\lambda_{3} u_{*}(T)=0$. If $\lambda_{3}<0$, then the function $\Phi(t)=\lambda_{3}-\lambda_{2}(t) G q_{*}(t)$ will be negative on some interval $(T-\varepsilon, T]$ and thus by the minimization condition (c) on the Hamiltonian the control must be given by $u_{*}(t)=a$ on this interval. Contradiction. Hence $\lambda_{3} \geq 0$.

Lemma 4.3. If $\lambda_{3}=0$, then the corresponding optimal control is constant over the interval $[0, T]$ and given by the control $u \equiv a$.

Proof. In this case the Hamiltonian function reduces to

$$
H=-\lambda_{1} \xi p \ln \left(\frac{p}{q}\right)+\lambda_{2}\left(b p-\left(\mu+d p^{\frac{2}{3}}\right) q-G u q\right)
$$

and thus the minimization condition (c) implies that

$$
u_{*}(t)=\left\{\begin{array}{ll}
0 & \text { if } \lambda_{2}(t)<0 \\
a & \text { if } \lambda_{2}(t)>0
\end{array} .\right.
$$

Since $\lambda_{2}(T)=0$ and $\dot{\lambda}_{2}(T)=-\xi \lambda_{1}(T) \frac{p_{*}(t)}{q_{*}(t)}=-\xi<0, \lambda_{2}$ is positive on some interval $(\tau, T]$ and here the control is given by $u_{*}(t)=a$. Since $p_{*}(T)=q_{*}(T)$, it follows that the trajectory entirely lies in $\mathcal{D}_{-}$as long as the control is $u \equiv a$. But then $\lambda_{2}$ cannot have another zero $\tau$ since otherwise $H(\tau)=-\lambda_{1}(\tau) \xi p(\tau) \ln \left(\frac{p(\tau)}{q(\tau)}\right) \neq 0$. Thus the control must be constant $u \equiv a$.

Except for this extremely degenerate case (the initial condition is such that with giving the full dose we reach the diagonal exactly when all inhibitors have been exhausted) we can, as we henceforth do, without loss of generality therefore assume that $\lambda_{3}$ is positive.

LEMma 4.4. If $\lambda_{3}>0$, then optimal controls end with an interval $(\tau, T]$ where $u_{*} \equiv 0$.

The function

$$
\Phi(t)=\lambda_{3}-\lambda_{2}(t) G q_{*}(t),
$$

which determines the structure of the optimal control $u_{*}$ through the minimization property (c) on the Hamiltonian $H$ is called the switching function of the problem and optimal controls satisfy

$$
u_{*}(t)=\left\{\begin{array}{ll}
0 & \text { if } \Phi(t)>0 \\
a & \text { if } \Phi(t)<0
\end{array} .\right.
$$

A priori the control is not determined by the minimum condition at times when $\Phi(t)=0$. If $\Phi(\tau)=0$, but $\dot{\Phi}(\tau) \neq 0$, then the control switches between $u=0$ and $u=a$ depending on the sign of $\dot{\Phi}(\tau)$. On the other hand, if $\Phi(t)$ vanishes identically on an open interval, then the minimization property in itself gives no information about the control. However, in this case also all derivatives of $\Phi(t)$ must vanish and this may and typically does determine the control. Controls of this kind are called singular while we refer to the constant controls as bang controls. Optimal controls then need to be synthesized from these candidates through an analysis of the switching function and its derivatives. 
The computations of the derivatives of the switching function $\Phi$ can be expressed concisely within the framework of geometric optimal control theory and we therefore now write the state as a 3-dimensional vector $z=\left(z_{1}, z_{2}, z_{3}\right)^{T}$ with $z_{1}=p, z_{2}=q$ and $z_{3}=y$. In vector notation the dynamics takes the form

$$
\dot{z}=f(z)+u g(z)
$$

with

$$
f(z)=\left(\begin{array}{c}
-\xi p \ln \left(\frac{p}{q}\right) \\
b p-\left(\mu+d p^{\frac{2}{3}}\right) q \\
0
\end{array}\right)
$$

and

$$
g(z)=\left(\begin{array}{c}
0 \\
-G q \\
1
\end{array}\right) .
$$

The adjoint equation then simply becomes

$$
\dot{\lambda}(t)=-\lambda(t)\left(D f(z(t))+u_{*}(t) D g(z(t))\right)
$$

where $D f$ and $D g$ denote the matrices of the partial derivatives of the vector fields which are evaluated along $z(t)$. The derivatives of the switching function can easily be computed using the following well-known result that can be verified by an elementary direct calculation. Here $\langle\cdot, \cdot\rangle$ denotes the standard inner product on $\mathbb{R}^{3}$, i.e. for a covector $\lambda \in\left(\mathbb{R}^{3}\right)^{*}$ and a vector $z \in \mathbb{R}^{3},\langle\lambda, z\rangle=\lambda z$.

Proposition 4.5. Let $h$ be a continuously differentiable vector field and define

$$
\Psi(t)=\langle\lambda(t), h(z(t))\rangle .
$$

Then the derivative of $\Psi$ along a solution to the system equation (4.8) for control $u$ and a solution $\lambda$ to the corresponding adjoint equation (4.11) is given by

$$
\dot{\Psi}(t)=\langle\lambda(t),[f+u g, h] z(t)\rangle,
$$

where

$$
[f, h](z)=D h(z) f(z)-D f(z) h(z)
$$

denotes the Lie bracket of the vector fields $f$ and $h$.

5. Synthesis of Optimal Controlled Trajectories. In this section we first give an overview of the structure of optimal controlled trajectories, but the proofs will be postponed to the remaining sections of the paper. We summarize the general structure of optimal controls and trajectories in the following theorem:

TheOREM 5.1. Given $\tilde{z}=(\tilde{p}, \tilde{q}, 0)$ with $(\tilde{p}, \tilde{q}) \in \mathcal{D}$, optimal controls are at most concatenations of the form $\mathbf{0}$ asa $\mathbf{0}$ with $\mathbf{0}$ denoting an arc along the constant control $u=0$, a denoting an arc along the constant control $u=a$, and $\mathbf{s}$ denoting an arc along the singular curve $\mathcal{S}$.

This result limits the possible concatenations in the sense that it provides an upper bound. But for many initial conditions the concatenation structure is quite 


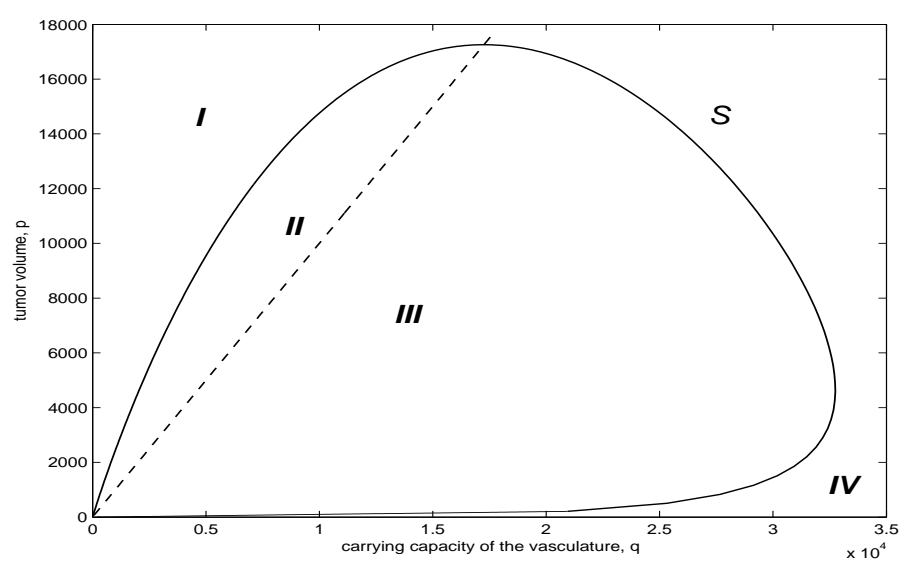

FIG. 5.1. The regions $I, I I, I I I$ and $I V$

shorter (pieces are missing) and there exists a unique extremal of this type which is the optimal solution. However, there also are initial conditions, for which there exists a 1-parameter family of extremals of this type and in these cases the optimal control needs to be computed numerically through minimizing a 1-dimensional function (see section 8). Once a simple maximal concatenation structure like the one given in Theorem 5.1 has been determined, this is a straightforward argument.

Clearly, optimal trajectories lie in $\mathbb{R}^{3}$ and at every point the actions depend on the available amount of inhibitors. However, it is more illustrative to consider the projections of trajectories into the $(p, q)$-plane ${ }^{1}$ and it is convenient, and only a slight abuse of terminology, not to distinguish in our language between the trajectories in $(p, q, y)$-space and their projections onto the $(p, q)$-coordinates.

The anchor piece of the synthesis is an optimal singular arc. It will be shown in section 6 that singular trajectories can only lie on a loop-like curve $\mathcal{S}$ in $(p, q)$-space where the vector fields $f$ and the Lie bracket $[f, g]$ are linearly dependent and that there exists a unique arc $\Gamma$ on $\mathcal{S}$ where the singular control is admissible, i.e. satisfies the control constraints $0 \leq u \leq a$. This curve $\mathcal{S}$ and the diagonal $\mathcal{D}_{0}=\{(p, q): p=q\}$ also form boundary curves between optimal bang-bang switchings in the order $a 0$ and of the reverse order $0 a$ and the concatenation structure of optimal controls is determined by the location of the initial condition relative to these curves. Denote by $\mathcal{S}_{+}$the region outside of the singular loop $\mathcal{S}$ and by $\mathcal{S}_{-}$the region inside this loop and define the following regions (see Figure 5.1)

$$
I=\mathcal{D}_{+} \cap \mathcal{S}_{+}, \quad I I=\mathcal{D}_{+} \cap \mathcal{S}_{-}, \quad I I I=\mathcal{D}_{-} \cap \mathcal{S}_{-}, \quad I V=\mathcal{D}_{-} \cap \mathcal{S}_{+} .
$$

In Figure 5.2 we indicate the structure of optimal controlled trajectories for a representative collection of initial conditions and have highlighted one example as a thick curve. (Pieces of trajectories corresponding to $u=a$ are shown as dashed curves and pieces of $u=0$ trajectories are shown as solid curves; pieces along the singular arc follow the curve $\mathcal{S}$.) The initial condition for the highlighted trajectory lies in region $I I$ and the optimal control is of the form as 0 : the control initially is given by $u=a$ until the singular $\operatorname{arc} \Gamma$ is reached; at this time the control switches to

\footnotetext{
${ }^{1}$ In our graphs we prefer to have $q$ as horizontal variable and $p$ along the vertical axis. Visually this better corresponds to a decrease or increase in the primary cancer volume.
} 


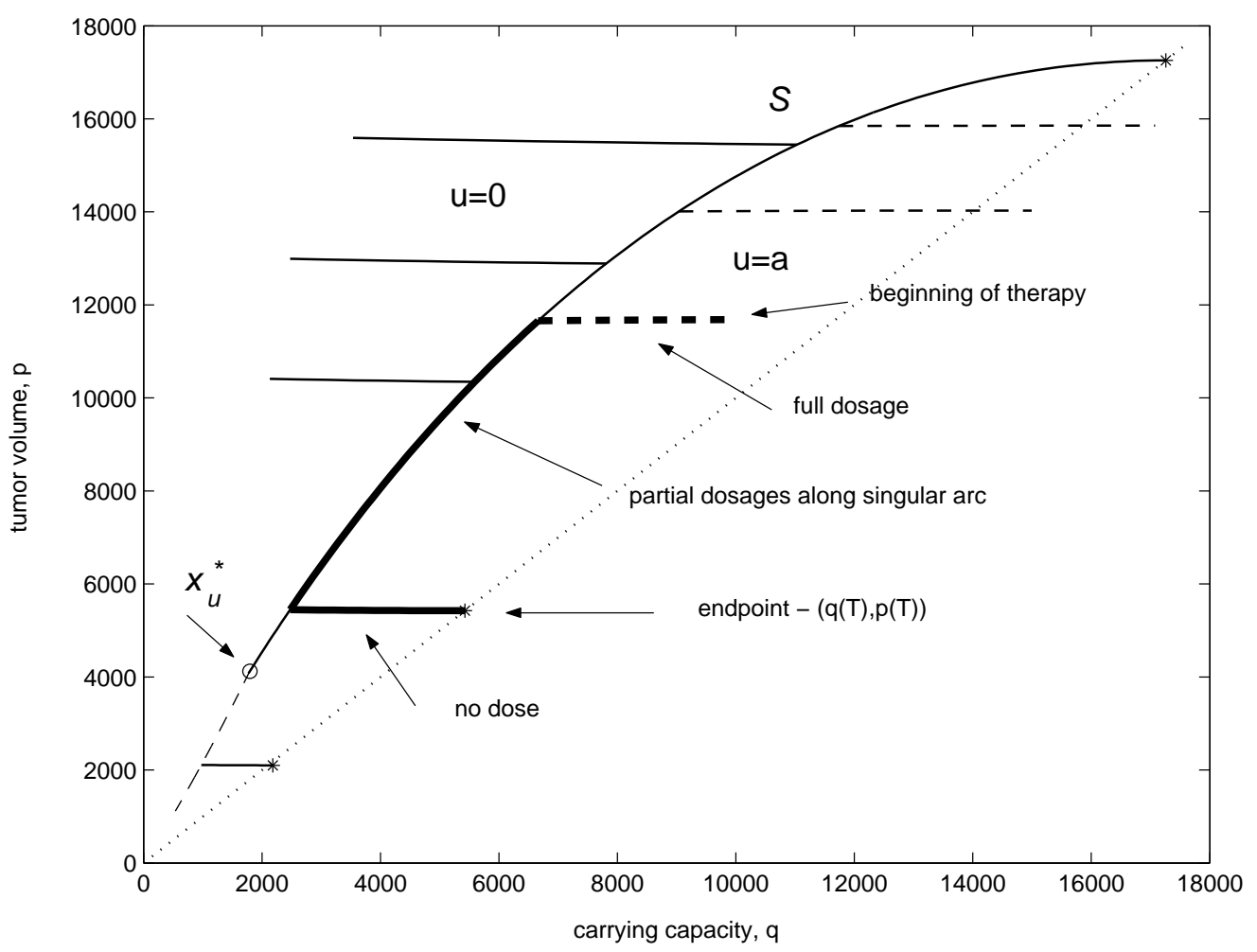

FIG. 5.2. Synthesis of optimal controlled trajectories

the singular control and follows the admissible singular arc $\Gamma$ until all inhibitors have been exhausted; due to after effects the minimum value for the tumor volume then is reached when, after termination of therapy, the system crosses the diagonal along the trajectory for $u=0$. This is the typical structure of optimal controlled trajectories for initial conditions in $I I$, but it depends on two facts: the overall amount of inhibitors is large enough to reach the singular arc, but it is not so large that the singular control would saturate along the singular arc at a specific point $x_{u}^{*}$ (computed in section 6.2 ). If there are not enough inhibitors to reach $\Gamma$, the optimal control is simple and is just of type $a 0$, giving all inhibitors at maximum dosage from the beginning until they become exhausted. If the amount of inhibitors is large enough so that the singular control would saturate at the point $x_{u}^{*}$ while following the singular arc, the structure of optimal controls is more complex and in this case controls can be of type $a 0$ or $a s a 0$. Typically, as the singular arc $\Gamma$ is reached, now the control switches and follows the singular arc for some time period, but in this case optimal trajectories leave the singular arc before reaching $x_{u}^{*}$ and the remaining inhibitors are exhausted along a full dose segment with $u=a$. But for trajectories that meet the singular arc close to the saturation point $x_{u}^{*}$ optimal controls do not switch to the singular control, but simply follow $u=a$ until inhibitors are exhausted. The precise structure of optimal trajectories that come close to the saturation point is rather difficult, but for a particular initial condition is easily resolved numerically.

For well-posed initial conditions in regions $I I I$ and $I V$, optimal controlled trajectories will eventually enter region $I I$ along a trajectory for $u=a$ and then follow 
the pattern described above. The specific form depends on the amount of inhibitors left over as the diagonal is crossed. For a typical initial condition, before crossing the diagonal $\mathcal{D}_{0}$, the control will simply be constant given by $u=a$ until region $I I$ is reached. Only for some initial conditions with a low $p$-value and a relatively high $q$-value the control can be $u=0$ initially and then will switch to $u=a$. For some points in $I V$ optimal controls could in principle have the full concatenation structure 0asa0, but these are not of separate interest for the underlying problem. (Essentially, following $u=0$ the trajectory enters the part of region $I I I$ where the control switches to $u=a$ and then ends with the pattern described for region $I I$.)

For initial conditions in region $I$ the most typical structure of optimal controls is $0 s 0$. Since $p>q$ the tumor is shrinking already, but as the system reaches the singular arc, it is best to administer therapy according to the singular control until all inhibitors become exhausted. As above, if the trajectory comes close to the saturation point, this structure changes into $0 s a 0$ and if the initial condition actually already is close to this point, it may simply be $a 0$ again.

A more precise description of all these possibilities is given in section 8 were we prove these results. Also, the diagrams shown here are generated using the parameter values given in section 2 that are taken from [9], but the qualitative structure of the solutions described here is robust with respect to parameter changes. Only if the upper limit $a$ on the dosage becomes too small, the singular arc will disappear.
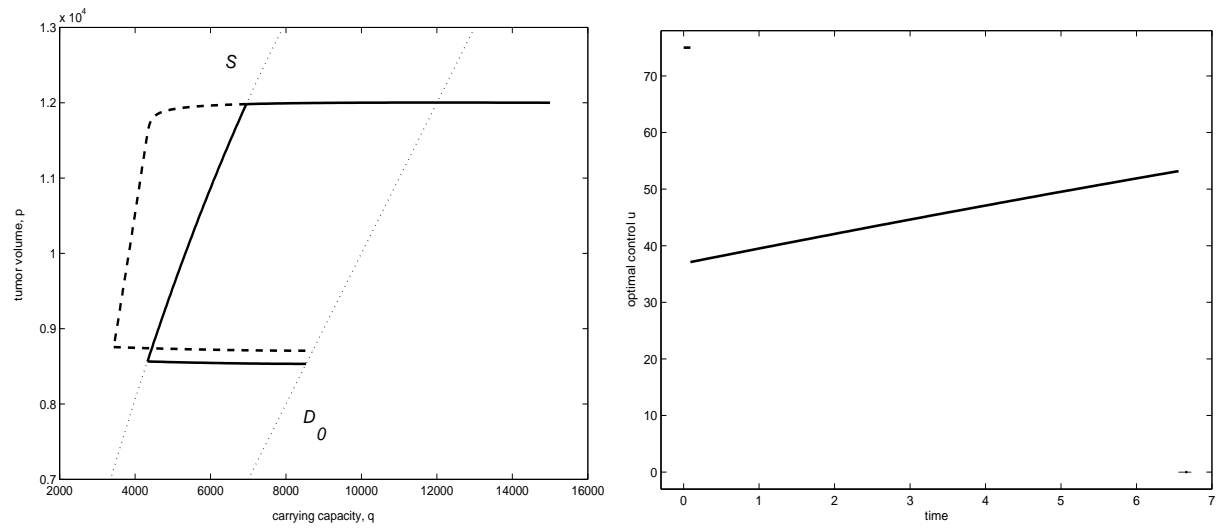

FIG. 5.3. An optimal as0-trajectory with corresponding control

In Figure 5.3 we give one specific example of an optimal trajectory (on the left) and its corresponding control (on the right) of the type as 0 . The initial condition is given by $(\tilde{p}, \tilde{q})=(12000,15000)$ in region $I I I$. The optimal control takes the maximal value $u=a$ for the short interval from 0 to $t_{1}=0.0905$ when the trajectory reaches the singular arc. At this point the control switches to the time-varying singular control until all inhibitors are being exhausted at time $t_{2}=6.5579$. Then, due to after effects, the minimum value of the tumor volume is realized a short period later at the final time $T=6.7221$ when the corresponding $u=0$ trajectory reaches the diagonal. Note the extremely fast $q$-dynamics away from the singular arc. The optimal final value is given by $p_{*}(T)=8533.4$. The optimal trajectory is shown as a solid curve in Fig. 5.3(a) and the singular curve $\mathcal{S}$ and the diagonal $\mathcal{D}_{0}$ are shown as dotted curves. For comparison we also show the $a 0$-trajectory that corresponds to the trajectory which applies all available inhibitors initially as dashed curve. (Its initial segment agrees 
with the optimal trajectory and is not marked separately.) This strategy leads to a tumor reduction with value 8707.4 at time 5.1934 .

6. Analysis of the Singular Arc. In this section we compute an explicit form for the singular control and the corresponding singular curve $\mathcal{S}$. We also show that there exists a unique connected $\operatorname{arc}$ of $\mathcal{S}$ where the singular control is admissible, i.e., satisfies the control constraints. Furthermore, the strengthened Legengre-Clebsch condition holds along $\mathcal{S}$ and thus the singular arc is locally optimal.

6.1. Computation of the singular control. Using Proposition 4.5 we get for the switching function $\Phi(t)=\langle\lambda(t), g(z(t))\rangle$ that

$$
\dot{\Phi}(t)=\langle\lambda(t),[f, g] z(t)\rangle
$$

and

$$
\ddot{\Phi}(t)=\langle\lambda(t),[f+u g,[f, g]] z(t)\rangle .
$$

Direct calculations verify that

$$
[f, g](z)=G p\left(\begin{array}{c}
\xi \\
-b \\
0
\end{array}\right)
$$

and

$$
[g,[f, g]](z)=-G^{2} b p\left(\begin{array}{l}
0 \\
1 \\
0
\end{array}\right)
$$

If the switching function $\Phi(t)=\lambda_{3}-\lambda_{2}(t) G q_{*}(t)$ vanishes at some time $t$, then $\lambda_{2}(t)$ is positive since $\lambda_{3}>0$ and thus we have

$$
\left.\langle\lambda(t),[g,[f, g]] z(t)\rangle=-\lambda_{2}(t) G^{2} d p_{*}(t)\right)<0,
$$

i.e., the so-called strengthened Legendre-Clebsch condition [11] is satisfied. Hence, and provided it is admissible, the singular control is of order 1, locally optimal, and given by

$$
u_{\sin }(t)=-\frac{\langle\lambda(t),[f,[f, g]] z(t)\rangle}{\langle\lambda(t),[g,[f, g]] z(t)\rangle} .
$$

Another direct computation verifies that

$$
[f,[f, g]](z)=G p\left(\xi b \ln \left(\frac{p}{q}\right)+\xi\left(\frac{2}{3} d \frac{\xi^{2}+\xi b \frac{p}{q}}{\sqrt[3]{p_{*}(t)}}-b\right)-\left(\mu+d p_{*}^{\frac{2}{3}}(t)\right) b\right)
$$

The vector fields $g,[f, g]$ and $[g,[f, g]]$ are everywhere linearly independent and thus $[f,[f, g]]$ can be expressed as a linear combination in this basis. In fact,

$$
[f,[f, g]]=\left(\xi+b \frac{p}{q}\right)[f, g]-\psi[g,[f, g]]
$$


with

$$
\psi=\psi(p, q)=\frac{1}{G}\left(\xi \ln \left(\frac{p}{q}\right)+b \frac{p}{q}+\frac{2}{3} \xi \frac{d}{b} \frac{q}{p^{\frac{1}{3}}}-\left(\mu+d p^{\frac{2}{3}}\right)\right) .
$$

Along a singular arc we have that

$$
\dot{\Phi}(t)=\langle\lambda(t),[f, g](z(t))\rangle \equiv 0
$$

and therefore

$$
\langle\lambda(t),[f,[f, g]](z(t))\rangle=-\psi(z(t))\langle\lambda(t),[g,[f, g]](z(t))\rangle \text {. }
$$

Since $\langle\lambda(t),[g,[f, g]](z(t))\rangle \neq 0$, the singular control defined by (6.6) is simply given by the function $\psi$ in feedback form, more precisely

$$
u_{\sin }(t)=-\frac{\langle\lambda(t),[f,[f, g]] z(t)\rangle}{\langle\lambda(t),[g,[f, g]] z(t)\rangle}=\psi(p(t), q(t)) .
$$

Summarizing, we have so far

Proposition 6.1. Let $\left(z_{*}, u_{*}\right)$ be an extremal pair. If the control $u_{*}$ is singular on an open interval $(\alpha, \beta)$, then $u_{*}$ is of order 1 and the strengthened Legendre-Clebsch condition is satisfied. The singular control is given in feedback form as

$$
\begin{aligned}
u_{\sin }(t) & =\psi\left(p_{*}(t), q_{*}(t)\right) \\
& =\frac{1}{G}\left(\xi \ln \left(\frac{p_{*}(t)}{\left.q_{*}(t)\right)}\right)+b \frac{p_{*}(t)}{\left.q_{*}(t)\right)}+\frac{2}{3} \xi \frac{d}{b} \frac{q_{*}(t)}{p_{*}^{\frac{1}{3}}(t)}-\left(\mu+d p_{*}^{\frac{2}{3}}(t)\right)\right)
\end{aligned}
$$

But note that the singular control is only admissible if this value lies in the interval $[0, a]$. Before addressing this issue, we first compute the singular curve itself.

6.2. Computation of the singular curve. For a trajectory to be an extremal, the singular curve also needs to satisfy the extra requirement that $H \equiv 0$, or, equivalently,

$$
\langle\lambda(t), f(z(t))\rangle \equiv 0
$$

Hence, along a singular arc, $\lambda(t)$ vanishes against the vector fields $f, g$ and $[f, g]$. Since $\lambda(t) \neq 0$, these vector fields must be linearly dependent. But $g$ is always linearly independent of $f$ and $[f, g]$ and thus the singular curve is precisely the locus where $f$ and $[f, g]$ are linearly dependent. Both vector fields do not depend on $y$ and have $y$-coordinate equal to 0 . With slight abuse of notation we can therefore also view them as vector fields on $(p, q)$-space and define the singular curve $\mathcal{S}$ as

$$
\mathcal{S}=\{(p, q): f(p, q) \wedge[f, g](p, q)=0\},
$$

where

$$
f(p, q) \wedge[f, g](p, q)=\left|\begin{array}{cc}
-\xi p \ln \left(\frac{p}{q}\right) & \xi \\
b p-\left(\mu+d p^{\frac{2}{3}}\right) q & -b
\end{array}\right|
$$


Thus the singular curve is given by the solutions of the equation

$$
\mu+d p^{\frac{2}{3}}=b \frac{p}{q}\left(1-\ln \left(\frac{p}{q}\right)\right) .
$$

The geometry of the singular curve becomes clear if we introduce a projective coordinate, i.e. make a blow-up in the variables of the form

$$
p=x q, \quad x>0
$$

The quotient $\frac{q}{p}$ is proportional to the endothelial density which is used to replace the carrying capacity of the vasculature as a variable in some models like, for example, in [7]. As it turns out, the singular curve and its corresponding singular control can be expressed solely in terms of the variable $x$ introduced here. This fact confirms mathematically the importance of this quantity. However, for the overall analysis, and in particular in view of a ready interpretation of the results, we preferred to keep the original variables $p$ and $q$ and only use $x$ in the analysis of the singular arc. In these variables equation (6.16) simplifies to

$$
\mu+d p^{\frac{2}{3}}=b x(1-\ln x)
$$

and can be rewritten in the form

$$
p^{2}+\varphi(x)^{3}=0
$$

with

$$
\varphi(x)=\frac{b x(\ln x-1)+\mu}{d}
$$

The function $\varphi$ is strictly convex with a minimum at $x=1$ and minimum value $\frac{\mu-b}{d}$. In particular, if $\mu \geq b$, then this equation has no positive solutions and thus no admissible singular arc exists. The case $\mu<b$, which we assumed in (3.5), is the medically relevant case. For $\mu=0$ the zeroes of $\varphi$ are given by $x_{1}^{*}=0$ and $x_{2}^{*}=e$ and $\varphi$ is negative on the interval $(0, e)$. In general, for $\mu>0$, we have $\varphi(0)=\frac{\mu}{d}=\varphi(e)$ and thus now the zeroes $x_{1}^{*}$ and $x_{2}^{*}$ satisfy $0<x_{1}^{*}<1<x_{2}^{*}<e$. We thus have

Proposition 6.2. The singular curve $\mathcal{S}$ entirely lies in the sector $\left\{(p, q): x_{1}^{*} q<\right.$ $\left.p<x_{2}^{*} q\right\}$ where $x_{1}^{*}$ and $x_{2}^{*}$ are the unique zeroes of the equation $\varphi(x)=0$ and satisfy $0 \leq x_{1}^{*}<1<x_{2}^{*} \leq e$. In the variables $(p, x)$ with $x=\frac{p}{q}$ the singular curve can be parameterized in the form

$$
p^{2}=\left(\frac{b x(1-\ln x)-\mu}{d}\right)^{3} \quad \text { for } \quad x_{1}^{*}<x<x_{2}^{*} .
$$

Proposition 6.3. Along the singular arc the singular control can be expressed solely as a function of $x$ in the form

$$
\Psi(x)=\frac{1}{G}\left[\left(\frac{1}{3} \xi+b x\right) \ln x+\frac{2}{3} \xi\left(1-\frac{\mu}{b x}\right)\right] .
$$

There exists exactly one connected arc on the singular curve $\mathcal{S}$ along which the control is admissible, i.e. satisfies the bounds $0 \leq \Psi \leq a$. This arc is defined over an interval 

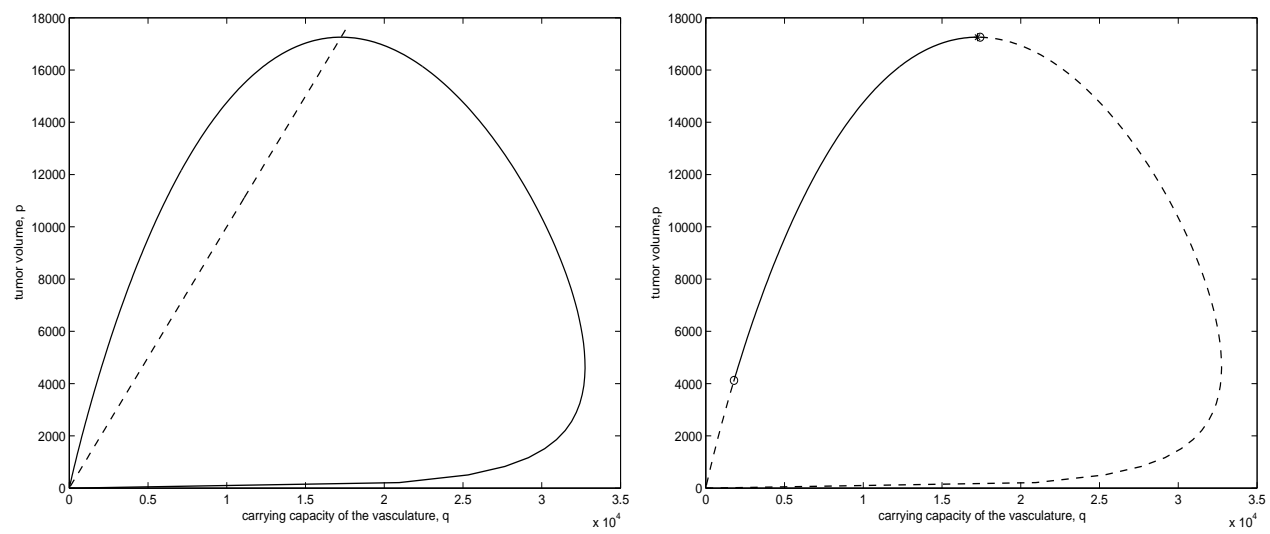

FIG. 6.1. The singular curve and its admissible part

$\left[x_{\ell}^{*}, x_{u}^{*}\right]$ where $x_{\ell}^{*}$ and $x_{u}^{*}$ are the unique solutions to the equations $\Psi\left(x_{\ell}^{*}\right)=0$ and $\Psi\left(x_{u}^{*}\right)=a$ and these values satisfy $x_{1}^{*}<x_{\ell}^{*}<x_{u}^{*}<x_{2}^{*}$.

Fig. 6.1 on the left gives a plot of the singular curve for the parameter values from [9] specified earlier and $\mu=0.02$ and shows the admissible portion of the petal like singular curve $\mathcal{S}$ for $a=75$ marked as a solid curve on the right. The qualitative structure shown in Fig. 6.1 is generally valid with the admissible portion shrinking for smaller values $a$.

Proof. In the variables $p$ and $x$ the singular control is given by

$$
u_{\sin }(t)=\frac{1}{G}\left(\xi \ln x(t)+b x(t)+\frac{2}{3} \xi \frac{d p(t)^{\frac{2}{3}}}{b x(t)}-\left(\mu+d p(t)^{\frac{2}{3}}\right)\right) .
$$

But along the singular arc we have $p^{\frac{2}{3}}=-\varphi(x)$ and thus we obtain the singular control as a feedback function of $x$ alone, $u_{\sin }(t)=\Psi(x(t))$, namely

$$
\begin{aligned}
\Psi(x) & =\frac{1}{G}\left(\xi \ln x+b x+\frac{2}{3} \xi \frac{b x(1-\ln x)-\mu}{b x}-b x(1-\ln x)\right) \\
& =\frac{1}{G}\left[\left(\frac{1}{3} \xi+b x\right) \ln x+\frac{2}{3} \xi\left(1-\frac{\mu}{b x}\right)\right] .
\end{aligned}
$$

Note that $\lim _{x \backslash 0} \Psi(x)=-\infty$ and $\lim _{x \rightarrow \infty} \Psi(x)=+\infty$. Now

$$
\begin{aligned}
\Psi^{\prime}(x) & =\frac{1}{G}\left[b(\ln x+1)+\frac{1}{3} \xi\left(\frac{1}{x}+2 \frac{\mu}{b x^{2}}\right)\right], \\
\Psi^{\prime \prime}(x) & =\frac{1}{G x^{3}}\left(b x^{2}-\frac{1}{3} \xi x-\frac{4}{3} \xi \frac{\mu}{b}\right),
\end{aligned}
$$

and the second derivative has a unique positive zero at

$$
x_{*}=\frac{1}{6} \frac{\xi}{b}\left(1+\sqrt{1+48 \frac{\mu}{\xi}}\right) .
$$

It follows that $\Psi$ is strictly concave for $0<x<x_{*}$ and strictly convex for $x>x_{*}$. If the function $\Psi$ has no stationary points, then $\Psi$ is strictly increasing and thus, as 
claimed, there exists a unique interval $\left[x_{\ell}^{*}, x_{u}^{*}\right]$ when $\Psi$ takes values in $[0, a]$ and the limits are the unique solutions of the equations $\Psi(x)=0$ and $\Psi(x)=a$, respectively. The same holds if $\Psi$ has a unique stationary point at $x_{*}$. In the remaining case, it follows from the convexity properties that $\Psi$ has a unique local maximum at $\tilde{x}_{1}<x_{*}$ and a unique local minimum at $\tilde{x}_{2}>x_{*}$. It suffices to show that $\Psi$ is negative at its local maximum. For this, as before, implies that $\Psi$ is strictly increasing when it is positive. Suppose now that $\Psi^{\prime}(\tilde{x})=0$. Then

$$
-b \ln \tilde{x}=b+\frac{1}{3} \xi\left(\frac{1}{\tilde{x}}+2 \frac{\mu}{b \tilde{x}^{2}}\right)>0
$$

and thus

$$
\begin{aligned}
\Psi(\tilde{x}) & =\frac{1}{G}\left[\left(\frac{1}{3} \xi+b \tilde{x}\right)\left(-1-\frac{1}{3} \frac{\xi}{b}\left(\frac{1}{\tilde{x}}+2 \frac{\mu}{b \tilde{x}^{2}}\right)\right)+\frac{2}{3} \xi\left(1-\frac{\mu}{b \tilde{x}}\right)\right] \\
& =\frac{1}{G}\left[-b \tilde{x}-\frac{1}{9} \frac{\xi^{2}}{b}\left(\frac{1}{\tilde{x}}+2 \frac{\mu}{b \tilde{x}^{2}}\right)-\frac{4}{3} \frac{\xi \mu}{b \tilde{x}}\right]<0 .
\end{aligned}
$$

Hence $\Psi$ is negative at any stationary point.

7. Analysis of Bang-Bang Junctions. Optimal controls are concatenations of the singular control with bang-bang structures and in this section we analyze possible switchings among bang-bang pieces of an optimal trajectory. We start with a strictly local analysis of switchings that establishes the regions in $(p, q)$-space where switchings from $u=a$ to $u=0$ or from $u=0$ to $u=a$ are possible. We then proceed to analyze extremal bang-bang concatenation structures over the full interval. These results will then be used in section 8 to determine the overall concatenation structures of optimal controls.

The singular curve computed in section 6.2 also is a boundary curve between optimal switchings in the order $a 0$ and of the reverse order $0 a$. Recall that $I=$ $\mathcal{D}_{+} \cap \mathcal{S}_{+}, I I=\mathcal{D}_{+} \cap \mathcal{S}_{-}, I I I=\mathcal{D}_{-} \cap \mathcal{S}_{-}$and $I V=\mathcal{D}_{-} \cap \mathcal{S}_{+}$.

PROPOSITION 7.1. Along optimal trajectories there are no switchings from $u=a$ to $u=0$ at points $(\tilde{p}, \tilde{q})$ in regions $I$ and $I I I$ and there are no switchings from $u=0$ to $u=a$ at points $(\tilde{p}, \tilde{q})$ in regions $I I$ and $I V$.

Proof. It follows from (4.7) that the derivative of the switching function must be non-positive at any time $\tau$ where the control switches from $u=0$ to $u=a$ and non-negative at every switching from $u=a$ to $u=0$. Furthermore, since $H \equiv 0$ along extremal lifts, at any switching $\tau$, the adjoint variable $\lambda(\tau)$ vanishes against both $f(z(\tau))$ and $g(z(\tau))$. Except for the points on the diagonal $\mathcal{D}_{0}=\{(p, q): p=q\}$, the vector fields $f, g$ and the coordinate vector field $\frac{\partial}{\partial y}=(0,0,1)^{T}$ are linearly independent and thus the Lie bracket $[f, g]$ can be written as a linear combination of these vector fields in the form

$$
[f, g](z)=\alpha(z) f(z)+\beta(z) g(z)+\gamma(z) \frac{\partial}{\partial y},
$$

i.e.,

$$
G p\left(\begin{array}{c}
\xi \\
-b \\
0
\end{array}\right)=\alpha(z)\left(\begin{array}{c}
-\xi p \ln \left(\frac{p}{q}\right) \\
b p-\left(\mu+d p^{\frac{2}{3}}\right) q \\
0
\end{array}\right)+\beta(z)\left(\begin{array}{c}
0 \\
-G q \\
1
\end{array}\right)+\gamma(z)\left(\begin{array}{c}
0 \\
0 \\
1
\end{array}\right)
$$


Thus we have

$$
\alpha(z)=-\frac{G}{\ln \left(\frac{p}{q}\right)}, \quad \beta(z)=\frac{b\left(\frac{p}{q}\right)\left(\ln \left(\frac{p}{q}\right)-1\right)+\left(\mu+d p^{\frac{2}{3}}\right)}{\ln \left(\frac{p}{q}\right)},
$$

and

$$
\gamma(z)=-\beta(z)
$$

At a switching time $\tau$,

$$
\begin{aligned}
\dot{\Phi}(\tau) & =\langle\lambda(\tau),[f, g](z(\tau))\rangle \\
& =\alpha(z)\langle\lambda(\tau), f(z(\tau))\rangle+\beta(z)\langle\lambda(\tau), g(z(\tau))\rangle-\beta(z) \lambda_{3} \\
& =-\beta(z) \lambda_{3}
\end{aligned}
$$

and by Lemma 4.3 we may assume that $\lambda_{3}$ is positive. Thus the sign of $\dot{\Phi}(\tau)$ is the opposite of the sign of $\beta$. The denominator of $\beta$ is positive in $\mathcal{D}_{+}$and negative in $\mathcal{D}_{-}$. The zero set of the numerator of $\beta$ is exactly the locus where the vector fields $f$ and $[f, g]$ are linearly dependent, i.e. the singular curve $\mathcal{S}$ (see (6.16)). We have labelled the regions so that the numerator is positive in $\mathcal{S}_{+}$and negative in $\mathcal{S}_{-}$(recall that $b>\mu$ ). Hence $\dot{\Phi}(\tau)$ is negative in regions $I$ and $I I I$ and positive in regions $I I$ and $I V$. This proves the proposition.

We now proceed to the analysis of bang-bang controls over the full interval. The admissible portion of the singular arc does not meet $\mathcal{D}_{-}$and in $\mathcal{D}_{-}$optimal controls will be bang-bang. We therefore begin with this analysis and assume as given a wellposed initial condition $\tilde{z}=(\tilde{p}, \tilde{q}, \tilde{y})$ with $(\tilde{p}, \tilde{q}) \in \mathcal{D}_{-}$. We first establish that optimal trajectories that start in $\mathcal{D}_{-}$will enter $\mathcal{D}_{+}$, but then cannot return to $\mathcal{D}_{-}$any more.

Proposition 7.2. Suppose $\left(p_{*}(\cdot), q_{*}(\cdot)\right)$ is an optimal trajectory defined over the interval $[0, T]$ with a well-posed initial condition $(\tilde{p}, \tilde{q}) \in \mathcal{D}_{-}$. Then there exists a time $\tau \in(0, T)$ so that the trajectory lies in $\mathcal{D}_{-}$for $t \in[0, \tau)$, crosses into $\mathcal{D}_{+}$at time $\tau$ and remains in $\mathcal{D}_{+}$for times $t \in(\tau, T)$. Over the interval $[0, \tau)$ the control either is constant given by $u \equiv a$ or it is of the form $0 a$. In the latter case the junction must lie in the set $\mathcal{N}_{-}=\left\{(p, q) \in \mathcal{D}_{-}: b p<\left(\mu+d p^{\frac{2}{3}}\right) q\right\}$.

Proof. Recall once more that we only consider initial conditions that are wellposed, i.e., the corresponding optimal trajectory does cross over into $\mathcal{D}_{+}$. Define $\tau$ as the (possibly) first time when the trajectory lies on the diagonal $\mathcal{D}_{0}$. We first show that $\tau$ cannot be a switching time. For, if this were the case, then, since $p(\tau)=q(\tau)$,

$$
H(\tau)=\lambda_{2}(\tau) p(\tau)\left(b-\left(\mu+d p(\tau)^{\frac{2}{3}}\right)\right)=0 .
$$

But on $\mathcal{D}_{0}$ we have $b>\mu+d p(\tau)^{\frac{2}{3}}$ and thus $\lambda_{2}(\tau)=0$. Hence the switching function at time $\tau$ is positive, $\Phi(\tau)=\lambda_{3}>0$. But then the control must be $u=0$ in a neighborhood of $\tau$ and the trajectory crosses from $\mathcal{D}_{+}$into $\mathcal{D}_{-}$. Contradiction.

Of the bang controls only $u=a$ steers the system from $\mathcal{D}_{-}$into $\mathcal{D}_{+}$, and nothing more needs to be shown about the interval $[0, \tau)$ if the control is constant and given by $u=a$ on this interval. If not, there exists a maximal interval $(\alpha, \beta)$ with $0<\alpha<$ $\tau<\beta<T$ so that the switching function is negative for $t \in(\alpha, \beta)$ and has zeroes at $\alpha$ and $\beta, \Phi(\alpha)=\Phi(\beta)=0$. The function $\Phi$ has a minimum over the interval $[\alpha, \beta]$ at some time $\sigma \in(\alpha, \beta)$ and by (6.1) and (6.3) we have

$$
\dot{\Phi}(\sigma)=G p(\sigma)\left(\xi \lambda_{1}(\sigma)-b \lambda_{2}(\sigma)\right)=0 .
$$


Hence $\lambda_{1}(\sigma)$ and $\lambda_{2}(\sigma)$ have the same sign. But $\lambda_{2}$ is positive along trajectories for $u=a$ since $\Phi(t)=\lambda_{3}-\lambda_{2}(t) G q(t)<0$ and $\lambda_{3}>0$. Hence $\lambda_{1}(\sigma)>0$. Since there is a junction at time $\alpha$, there also exists an interval $(\alpha-\varepsilon, \alpha)$ where the control is $u=0$ and on this interval we have

$$
H(t)=-\lambda_{1}(t) \xi p(t) \ln \left(\frac{p(t)}{q(t)}\right)+\lambda_{2}(t)\left(b p(t)-\left(\mu+d p(t)^{\frac{2}{3}}\right) q(t)\right)=0
$$

while $\lambda_{2}$ is still positive for $\varepsilon$ small. If we have

$$
b p(\alpha)>\left(\mu+d p(\alpha)^{\frac{2}{3}}\right) q(\alpha)
$$

at the junction, then, by (7.4), $\lambda_{1}$ must be negative on this interval and also $\lambda_{1}(\alpha)<0$. Hence there exists a last zero for $\lambda_{1}$ in the interval $(\alpha, \sigma)$, say $\lambda_{1}(\rho)=0$. At this zero the adjoint equation (4.2) reads

$$
\dot{\lambda}_{1}(\rho)=\lambda_{2}(\rho)\left(\frac{2}{3} d \frac{q(\rho)}{\sqrt[3]{p(\rho)}}-b\right)
$$

The curve $\mathcal{N}_{0}=\left\{(p, q): b p=\left(\mu+d p^{\frac{2}{3}}\right) q\right\}$ is the $\dot{q}$-nullcline for $u=0$ and since at the points on the $\dot{q}$-nullcline we have

$$
f(z)=\left(\begin{array}{c}
-\xi p \ln \left(\frac{p}{q}\right) \\
0 \\
0
\end{array}\right)
$$

with the first coordinate positive in $\mathcal{D}_{-}$, corresponding trajectories cross $\mathcal{N}_{0}$ from $\mathcal{N}_{-}=\left\{(p, q): b p<\left(\mu+d p^{\frac{2}{3}}\right) q\right\}$ into $\mathcal{N}_{+}=\left\{(p, q): b p>\left(\mu+d p^{\frac{2}{3}}\right) q\right\}$ (Fig. 7.1).

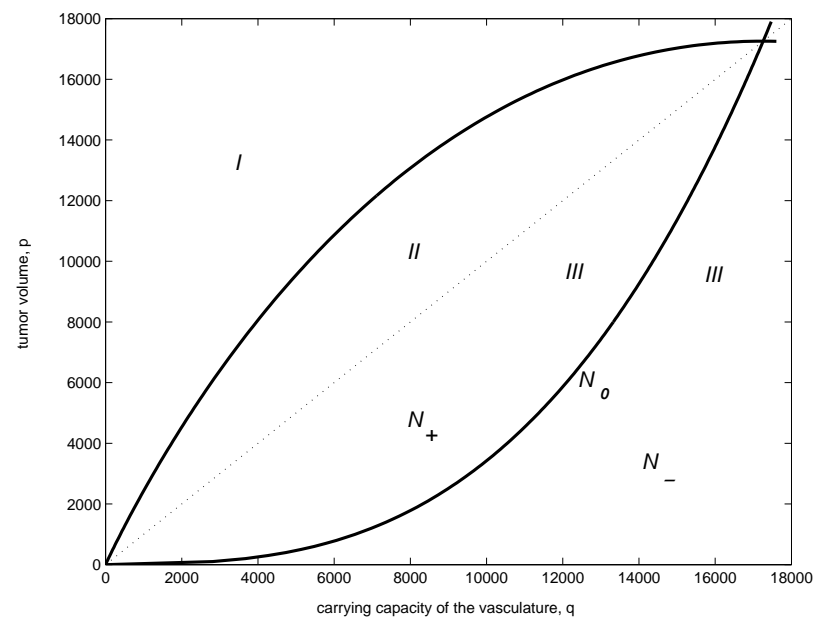

FIG. 7.1. The curve $\mathcal{N}_{0}$

Because of the extra control term $-G q a$ in the vector field $g$, this also holds for trajectories corresponding to the control $u=a$. Hence, if the junction at time $\alpha$ satisfies (7.5), then so does the arc forward in time, i.e. for all $t \in(\alpha, \sigma)$ we have

$$
q(t)<\frac{b p(t)}{\mu+d p(t)^{\frac{2}{3}}}
$$


Hence

$$
\frac{2}{3} d \frac{q(\rho)}{\sqrt[3]{p(\rho)}}-b<\frac{2}{3} d \frac{b p(\rho)^{\frac{2}{3}}}{\mu+d p(\rho)^{\frac{2}{3}}}-b=\frac{2}{3} b \frac{d p(\rho)^{\frac{2}{3}}}{\mu+d p(\rho)^{\frac{2}{3}}}-b<-\frac{1}{3} b<0
$$

Since the multiplier $\lambda_{2}$ is positive along $u=a$ we therefore have $\dot{\lambda}_{1}(\rho)<0$. Contradiction. Thus no $0 a$-junction can lie in the interior of $\mathcal{N}_{+}$. The same reasoning also precludes that $0 a$-junctions would lie on the $\dot{q}$-nullcline for $u=0$, i.e. on $\mathcal{N}_{0}$. In this case it follows that $\lambda_{1}(\alpha)=0$ and $\dot{\lambda}_{1}(\alpha)<0$, and thus again there still exists a last zero of $\lambda_{1}$ in the interval $(\alpha, \sigma)$ where the same contradiction arises. Thus, any possible $0 a$-junction in $\mathcal{D}_{-}$must lie in $\mathcal{N}_{-}$.

We now show that, if there is a junction at some time $\alpha$, then the control must be constant $u=0$ on the initial interval $[0, \alpha)$. Since trajectories for $u=0$ cross $\mathcal{N}_{0}$ from $\mathcal{N}_{-}$into $\mathcal{N}_{+}$, it follows that as long as the control $u=0$ is used for $t<\alpha$, the trajectory lies in $\mathcal{N}_{-}$. Since this trajectory also necessarily lies in $\mathcal{D}_{-}$we have $p(t)<q(t)$. But then the identity

$$
H(t)=-\lambda_{1}(t) \xi p(t) \ln \left(\frac{p(t)}{q(t)}\right)+\lambda_{2}(t)\left(b p(t)-\left(\mu+d p(t)^{\frac{2}{3}}\right) q(t)\right)=0
$$

implies that neither $\lambda_{1}$ nor $\lambda_{2}$ can have any zeroes (otherwise they would need to vanish simultaneously contradicting Lemma 4.2). Hence $\lambda_{2}$ is positive and $\lambda_{1}$ must be negative as long as the control is $u=0$. This precludes any more switchings. For, if there is another switching $0<\theta<\alpha$, then there also needs to be another zero for the derivative of the switching function in $(\theta, \alpha)$ and at this derivative $\lambda_{1}$ and $\lambda_{2}$ must have the same sign.

Thus, if $(\tilde{p}, \tilde{q}) \in \mathcal{D}_{-}$is a well-posed initial condition, then there exists a first time $\tau$ at which the trajectory crosses from $\mathcal{D}_{-}$into $\mathcal{D}_{+}$and the control over $[0, \tau)$ is either constant and given by $u \equiv a$ or it has exactly one switching from 0 to $a$ with the junction in $\mathcal{N}_{-}$. It still remains to show that the trajectory cannot return from $\mathcal{D}_{+}$ into $\mathcal{D}_{-}$for times $t>\tau$, i.e. that $(p(t), q(t))$ lies in $\mathcal{D}_{+}$for $t \in(\tau, T)$. (It follows from Lemma 4.1 that the endpoint lies on $\mathcal{D}_{0}$.)

If the trajectory were to return to $\mathcal{D}_{-}$, then there would exist another time $\kappa>\beta$ where the trajectory again would cross $\mathcal{D}_{0}$ with control $u=0$ in a neighborhood of $\kappa$. Now (7.10) implies that $\lambda_{2}(\kappa)=0$ and thus the adjoint equation for $\lambda_{2}$ gives $\dot{\lambda}_{2}(\kappa)=-\xi \lambda_{1}(\kappa)$. Since $\lambda_{1}$ and $\lambda_{2}$ cannot vanish simultaneously, we have $\lambda_{1}(\kappa) \neq 0$ and $\lambda_{2}$ changes sign at $\tau$. If $\lambda_{1}(\kappa)>0$, then $\lambda_{2}$ is negative for $t>\tau$, $t$ near $\tau$. However, after crossing into $\mathcal{D}_{-}$, trajectories for $u=0$ entirely lie in $\mathcal{D}_{-}$and do not cross back into $\mathcal{D}_{+}$. Consequently it follows from (7.10) that $\lambda_{2}$ cannot have another zero along an $u=0$ arc. (In $\mathcal{D}_{-}$the expression $\lambda_{1} \xi p \ln \left(\frac{p}{q}\right)$ can only vanish if $\lambda_{1}=0$ and this precludes $\lambda_{2}$ from having a zero.) But then the switching function $\Phi(t)=\lambda_{3}-\lambda_{2}(t) G q$ remains positive and so there cannot be another switching in the control. But this structure clearly is not optimal since the value for $p$ increases in $\mathcal{D}_{-}$. Contradiction. If $\lambda_{1}(\kappa)<0$, we are back in the situation considered above: if all inhibitors have not been used up, there needs to exist another switching to $u=a$; but the entire forward orbit of the $u=0$ trajectory lies in $\mathcal{N}_{+}$and therefore this switching would lie in $\mathcal{N}_{+}$as well violating the earlier statement.

This proposition implies that for well-posed initial conditions $(\tilde{p}, \tilde{q}, 0)$ with $(\tilde{p}, \tilde{q}) \in$ $\mathcal{N}_{+}$the optimal control is given in feedback form by $u \equiv a$ until the trajectory crosses over into $\mathcal{D}_{+}$at time $\tau$. The further structure of the optimal control depends on 
the amount of inhibitors $y(\tau)$ that are still available at this time. If this amount is too small to reach the singular arc, then, since only junctions in the order $a 0$ are optimal in region $I I=\mathcal{D}_{+} \cap \mathcal{S}_{-}$, the optimal control is simply given by $u=a$ until all inhibitors are exhausted and then follows $u=0$ until the trajectory terminates on the diagonal $\mathcal{D}_{0}$. (If the control would switch prior to the time when all inhibitors are exhausted, by Proposition 7.1 it cannot switch back to the control $u=a$ in region $I I$ and thus would reach the diagonal with inhibitors still available contradicting Lemma 4.1.) Hence, if enough inhibitors are available for the trajectory to reach the singular curve $\mathcal{S}$, then the trajectory will follow $u=a$ in region $I I$.

We now consider the further structure of optimal controls for segments of the trajectory that lie in $\mathcal{D}_{+}$. We first show that segments corresponding to the control $u=0$ can only lie at the beginning or at the end of the interval $[0, T]$.

Proposition 7.3. Let $(\alpha, \beta)$ be a maximal open interval where the optimal control is given by $u \equiv 0$ with corresponding trajectory $\left(p_{*}, q_{*}\right)$ lying in $\mathcal{D}_{+}$. Then $\alpha$ and $\beta$ cannot both be switching times. If $\alpha$ is a switching time, then $\beta=T$, the final time, and if $\beta$ is a switching time, then $\alpha=0$, the initial time.

Proof. As before, on the interval $(\alpha, \beta)$ equation (7.10) holds and on $\mathcal{D}_{+}$we have $\xi p \ln \left(\frac{p}{q}\right)>0$ and $b p-\left(\mu+d p^{\frac{2}{3}}\right) q>0$. As above, in this case neither $\lambda_{1}$ nor $\lambda_{2}$ can vanish and therefore $\lambda_{1}$ and $\lambda_{2}$ have the same sign over $(\alpha, \beta)$. Since $\lambda_{2}$ is positive at switchings times, it follows that both $\lambda_{1}$ and $\lambda_{2}$ are positive over $[\alpha, \beta]$ if at least one of the endpoints is a switching time. Along $u=0$ the derivatives of the switching function are given by $\dot{\Phi}(t)=\langle\lambda(t),[f, g](z(t))\rangle$ and $\ddot{\Phi}(t)=\langle\lambda(t),[f,[f, g]](z(t))\rangle$. If there exists a time $\tau \in(\alpha, \beta)$ where $\dot{\Phi}(\tau)=0$, then it follows from (6.3) and (6.8) that

$$
\begin{aligned}
\ddot{\Phi}(\tau) & =\left(\xi+b \frac{p(\tau)}{q(\tau)}\right)\langle\lambda(\tau),[f, g](z(\tau))\rangle-\psi(p(\tau), q(\tau))\langle\lambda(\tau),[g,[f, g]](z(\tau))\rangle \\
& =\left(\xi+b \frac{p(\tau)}{q(\tau)}\right) \dot{\Phi}(\tau)-\psi(p(\tau), q(\tau))\langle\lambda(\tau),[g,[f, g]](z(\tau))\rangle \\
& =\psi(p(\tau), q(\tau)) b G^{2} p(\tau) \lambda_{2}(\tau)>0
\end{aligned}
$$

Here we use that $\psi$ is positive in $\mathcal{D}_{+}$.

Suppose $\alpha$ is a switching time. Then there exists an $\varepsilon>0$ so that $\dot{\Phi}$ is positive in $(\alpha, \alpha+\varepsilon)$. (Since the control is $u=0$, we have $\dot{\Phi}(\tau) \geq 0$, and even if $\dot{\Phi}(\alpha)=0$, then this is implied by $\ddot{\Phi}(\alpha)>0$.) Thus, if $\dot{\Phi}$ has zeroes in $(\alpha, \beta)$, then there exists a smallest one, call it $\tau$. But then $\dot{\Phi}(t)>0$ on the interval $(\alpha, \tau)$ and so $\Phi$ cannot have a local minimum at $\tau$ contradicting $\ddot{\Phi}(\tau)>0$. Hence $\Phi$ is strictly increasing over $(\alpha, \beta)$ as long as the control $u=0$ is used and there cannot be another zero at $\beta$. Similarly, if $\beta$ is a switching time, then $\Phi$ is strictly decreasing over $(\alpha, \beta)$ as long as the control $u=0$ is used and again there cannot exist a previous zero at $\alpha$.

Proposition 7.4. Suppose $\left(p_{*}, q_{*}\right)$ is an optimal trajectory corresponding to the constant control $u=a$ over some open interval $(\alpha, \beta)$ with switching times at $\alpha$ and $\beta$. Then $(p(\alpha), q(\alpha)) \notin I I$ and $(p(\beta), q(\beta)) \in I I$. Furthermore, there exists a time $\tau \in(\alpha, \beta)$ where $\psi(p(t), q(t)) \geq a$.

Proof. The statements about the junction points follow from Proposition 7.1. Along $u=a$ the switching function is negative over $(\alpha, \beta)$ and has a minimum at some time $\tau \in(\alpha, \beta)$ where $\dot{\Phi}(\tau)=0$ and $\ddot{\Phi}(\tau) \geq 0$; and the derivatives of the switching function are now given by $\dot{\Phi}(t)=\langle\lambda(t),[f, g](z(t))\rangle$ and

$$
\ddot{\Phi}(t)=\langle\lambda(t),[f+a g,[f, g]](z(t))\rangle \text {. }
$$


As above, it follows from (6.3) and (6.8) that

$$
\begin{aligned}
\ddot{\Phi}(\tau) & =\left(\xi+b \frac{p(\tau)}{q(\tau)}\right)\langle\lambda(\tau),[f, g](z(\tau))\rangle+\{a-\psi(p(\tau), q(\tau))\}\langle\lambda(\tau),[g,[f, g]](z(\tau))\rangle \\
& =\left(\xi+b \frac{p(\tau)}{q(\tau)}\right) \dot{\Phi}(\tau)+\{a-\psi(p(\tau), q(\tau))\}\langle\lambda(\tau),[g,[f, g]](z(\tau))\rangle \\
& =\{\psi(p(\tau), q(\tau))-a\} b G^{2} p(\tau) \lambda_{2}(\tau) .
\end{aligned}
$$

Since $\Phi(\tau)=\lambda_{3}-\lambda_{2}(\tau) \operatorname{Gaq}(\tau)<0$, we have $\lambda_{2}(\tau)>0$ and thus we must have $\psi(p(\tau), q(\tau)) \geq a$. This proves the result.

8. Synthesis of Optimal Controls. We now put together the results and consider concatenations between singular and bang arcs and prove the results of section 5. We start with a strictly local analysis of singular junctions analogous to Proposition 7.1 for bang-bang junctions and show that all possible concatenations of bang controls with the singular arc are extremal. This classical result is included for sake of completeness and is a direct consequence of the fact that the strengthened LegendreClebsch condition is satisfied. We then show that extremals which contain a saturating arc are not optimal and proceed to the analysis of the possible concatenations of bang and singular arcs.

Proposition 8.1. Let I be an open interval on which the optimal control $u_{*}$ is singular and takes values in the interior of the control set. Then concatenations of both the forms $\mathbf{b s}$ and $\mathbf{s b}$ where $\mathbf{b}$ stands for any of the two bang controls $u=0$ or $u=a$ are extremal.

Proof. Recall that for any control $u$ that is continuous from the left (-) or right $(+)$ the second derivative of the switching function is given by

$$
\ddot{\Phi}(t \pm)=\langle\lambda(t),[f,[f, g]](z(t))\rangle+u(t \pm)\langle\lambda(t),[g,[f, g]](z(t))\rangle
$$

and it vanishes identically on $I$ along the singular control. Since the strengthened Legendre-Clebsch condition is satisfied, we have $\langle\lambda(t),[g,[f, g]](z(t))\rangle<0$. By assumption the singular control takes values in in the interior of the control set $[0, a]$ and thus $\langle\lambda(t),[f,[f, g]](z(t))\rangle>0$. Hence, for $u=0$ we get $\ddot{\Phi}(t)>0$ and for $u=a$ we have $\ddot{\Phi}(t)<0$. These signs are consistent with entry and exit from the singular arc for each control, i.e., for example, if $u=0$ on an interval $(\tau-\varepsilon, \tau)$, then $\Phi$ is positive over this interval consistent with the choice $u=0$ as minimizing control.

Thus, as long as the singular control has not saturated, it is possible to jump onto or off the singular arc with the constant controls $u=0$ or $u=a$ at any point without violating the conditions of the Maximum Principle locally. Naturally, optimality over longer time-intervals is not guaranteed and still needs to be analyzed. As an example, it follows from Proposition 7.3 that the singular arc can only be left with the control $u=0$ when all inhibitors have been exhausted. On the other hand, trajectories for $u=a$ can (and sometimes must) leave the singular arc before all inhibitors have been exhausted. This follows from the result below. Recall that $x_{u}^{*}$ is the point introduced in Proposition 6.3 where the singular control saturates at the upper control value $a$.

Proposition 8.2. At the saturation point $x_{u}^{*}$ on the singular arc where the singular control saturates at the upper value $u=a$, it is not optimal to continue the control with $u=a$. Thus optimal trajectories need to leave the singular arc before saturation.

This result may seem somewhat counterintuitive, but this is indeed the typical behavior at saturation in low dimensions (see, for example, [17] or [2]). 
Proof. Consider the trajectory that follows the singular arc and at the saturation time $\tau$ continues with the control $u=a$. In general, we have

$$
\ddot{\Phi}(t)=\left(\xi+b \frac{p(t)}{q(t)}\right) \dot{\Phi}(t)+(u(t)-\psi(p(t), q(t)))\langle\lambda(t),[g,[f, g]](z(t))\rangle .
$$

Along the singular arc $\dot{\Phi}(\tau)=0$ and at the saturation point we also have $\ddot{\Phi}(\tau)=0$ for the control $u=a$ since $\psi=a$. Hence, along $u=a$ we get from the right that

$$
\begin{aligned}
\Phi^{(3)}(\tau+) & =-\left(\frac{d}{d t}_{\mid t=\tau} \psi(p(t), q(t))\right)\langle\lambda(t),[g,[f, g]](z(t))\rangle \\
& =\left(\frac{d}{d t}_{\mid t=\tau} \psi(p(t), q(t))\right) b G^{2} \lambda_{2}(\tau) p(\tau) .
\end{aligned}
$$

Recall that $\psi(p(t), q(t))=\Psi(x(t))$ with $x=\frac{p}{q}$ and $\Psi$ defined in (6.22) in Proposition 6.3. We thus have

$$
\frac{d}{d t}_{\mid t=\tau} \psi(p(t), q(t))=\Psi^{\prime}\left(x_{u}^{*}\right) \dot{x}(\tau)
$$

It follows from the proof of Proposition 6.3 that $\Psi^{\prime}\left(x_{u}^{*}\right)>0$ and in general we have

$$
\dot{x}=\frac{\dot{p} q-p \dot{q}}{q^{2}}=-\xi x \ln x-b x^{2}+\left(\mu+d p^{\frac{2}{3}}\right) x+G u x .
$$

Substituting $\left(\mu+d p^{\frac{2}{3}}\right)=b x(1-\ln x)$ along the singular arc (c.f. (6.18)), we get

$$
\dot{x}=x(G u-(\xi+b x) \ln x) .
$$

But at the saturation point we also have

$$
G u(\tau)=G a=\left(\frac{1}{3} \xi+b x(\tau)\right) \ln x(\tau)+\frac{2}{3} \xi\left(1-\frac{\mu}{b x(\tau)}\right)
$$

and thus

$$
\dot{x}(\tau)=\frac{2}{3} \xi\left(x(\tau)(1-\ln x(\tau))-\frac{\mu}{b}\right)=\frac{2}{3} \frac{\xi}{b} d p(\tau)^{\frac{2}{3}}>0
$$

Hence

$$
\Phi^{(3)}(\tau+)>0
$$

and $\Phi$ is positive for $t>\tau, t$ near $\tau$, contradicting the minimization property for $u=a$.

Thus optimal trajectories cannot continue with the saturated control $u=a$ after the saturation point, but instead must leave the singular arc prior to saturating. The analogous computation with $u=0$ for $t>\tau$ shows that we can switch to $u=0$ at saturation, but by Proposition 7.3 this is only optimal if all inhibitors have been exhausted. In general, if inhibitors are available to go beyond the saturation point, optimal trajectories must leave the singular arc before saturation occurs. When precisely this happens, depends on the amount of inhibitors left. For example, it is clear that if $\tilde{z}=(\tilde{p}, \tilde{q}, \tilde{y})$ is a point with $(\tilde{p}, \tilde{q}) \in \mathcal{S}$ before saturation for which $A-\tilde{y}$ is small, then it is not optimal to leave the singular arc simply because there are not 
enough inhibitors left so that the system could reach the region $I I=\mathcal{D}_{+} \cap \mathcal{S}_{-}$where a switching from $u=a$ to $u=0$ again is optimal. In this case optimal trajectories follow the singular arc until inhibitors are exhausted. However, if enough inhibitors are available so that the singular arc would lose admissibility before they all are used up, then indeed by leaving the singular arc earlier trajectories can enter region $I I$ and this will be the optimal strategy. But now the argument needs to become global. Since by now we have sufficiently reduced the possibilities in the structures of optimal controls and trajectories, we can reduce this problem to a 1-dimensional optimization problem that can easily be solved numerically. Based on our previous analysis of the structure of extremals, we now determine the optimal synthesis on $\mathcal{D}$. We not only establish the qualitative structure claimed in Theorem 5.1, but also show how to compute the optimal control for a given initial condition $\tilde{z}=(\tilde{p}, \tilde{q}, 0)$.

We will prove that except for some initial conditions $(\tilde{p}, \tilde{q})$ in regions $I I I$ and $I V$ (which are less relevant for the underlying problem) our local analysis above only allows for at most a 1-parameter family of extremals $\Gamma_{\varepsilon}(s)=\left(p_{\varepsilon}(s), q_{\varepsilon}(s), y_{\varepsilon}(s)\right)$, $0 \leq s \leq T(\varepsilon)$, with the parameter $\varepsilon$ ranging over a compact interval $I=[0, \theta]$. The corresponding value of the objective is given by $v(\varepsilon), v(\varepsilon)=p_{\varepsilon}(T(\varepsilon))$ and it will be clear from the definition of the family $\Gamma_{\varepsilon}$ that the value $v(\varepsilon)$ depends continuously on $\varepsilon$. Thus, for every initial condition there exists an optimal control that is determined by numerical minimization of $v(\varepsilon)$ over $[0, \theta]$. However, the structure of this one-parameter family of extremals depends on the location of $(\tilde{p}, \tilde{q})$ and we need to distinguish three cases.

(1) We start with an initial condition $(\tilde{p}, \tilde{q})$ in region $I$ and will show that in this case optimal controls at most have the form 0sa0 (possibly with the initial 0s sequence absent). Let $\varsigma_{+}$denote the reference trajectory that starts at $\tilde{z}=(\tilde{p}, \tilde{q}, 0)$, uses the control $u=0$ until the singular arc is reached at some time $\tau$ (the existence of such a time is clear for initial conditions of this type) and then follows the singular arc for time $\sigma$ until either all available inhibitors have been exhausted or the saturation point is reached. We now use the time $\varepsilon$ along this trajectory as parameter and construct the family $\Gamma_{\varepsilon}$ over the compact parameter interval $[0, \theta]$ with $\theta=\tau+\sigma$ as follows: the trajectory $\Gamma_{\varepsilon}(\cdot)$ agrees with the reference trajectory $\varsigma_{+}$up to time $\varepsilon$, switches at time $\varepsilon$ to the control $u=a$ which will then be followed until all remaining inhibitors have been exhausted, and then the control still is $u=0$ until the trajectory terminates at time $T$ as the diagonal is reached.

This family indeed contains all possible extremals starting at initial condition $\tilde{z}$ : For, initially the control can only be $u=0$ or $u=a$. If the control is $u=a$, then it follows from the phase portrait for $u=a$ that this trajectory does not meet the admissible portion of the singular curve. Since any possible junction will lie in $\mathcal{D}_{+}$, it follows from Proposition 7.3 that the control can only switch to $u=0$ as all inhibitors have been exhausted. At this point the optimal control then is still given by $u=0$ until the diagonal $\mathcal{D}_{0}$ is reached at time $T(0)$ and the trajectory is terminated. This is the trajectory $\Gamma_{0}(\cdot)$ in our family. It actually follows from Proposition 7.1 that this trajectory would not be an extremal if the switching were to lie in region $I$ and in this case this trajectory could be excluded a priori. However, even if this is the case, for simplicity of argument we retain this curve anyway. Similarly, if initially the control is $u=0$ on $[0, \varepsilon]$ with $\varepsilon \leq \tau$ and then switches to $u=a$, the same reasoning applies and the structure of the corresponding control is simply $0 a 0$. For $\varepsilon>\tau$, the trajectory $\Gamma_{\varepsilon}(\cdot)$ now follows the control $u=0$ until time $\tau$, then stays on the singular arc until time $\varepsilon$ at which time it leaves the singular arc to use $u=a$ until all inhibitors are 


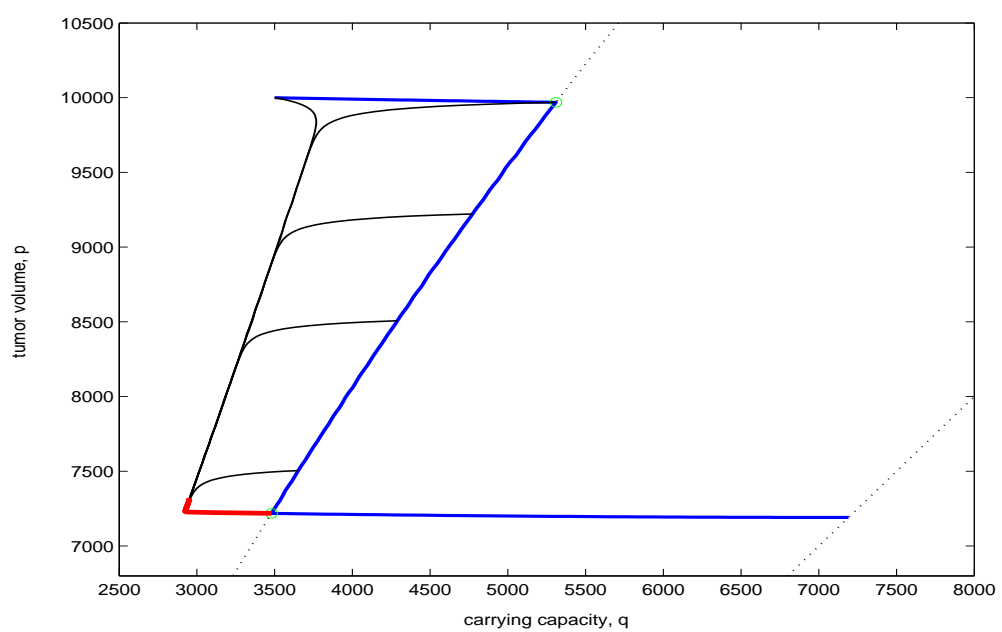

FIG. 8.1. Parameterized extremals for an initial condition in region I without saturation

being exhausted. The remaining time along the $a$-trajectory is given by

$$
\eta(\varepsilon)=\frac{1}{a}\left(A-\int_{\tau}^{\varepsilon} \psi\left(p_{\varepsilon}(s), q_{\varepsilon}(s)\right) d s\right)
$$

with $\eta(\tau+\theta)=0$ if the inhibitors get exhausted along the singular arc. If the singular control saturates, we know that the trajectory $\Gamma_{\theta}(\cdot)$ is no longer optimal and therefore can terminate the construction of the 1-parameter family of extremals with the trajectory that switches to $u=a$ at saturation. This simply provides us with a compact parameter interval, but in this case the minimum will be attained at a parameter $\varepsilon<\theta$. It follows from our local analysis given before that any possible extremal that could start at $\tilde{z}$ is part of this family $\Gamma_{\varepsilon}(\cdot)$. Essentially, trajectories cannot switch to $u=0$ before all inhibitors have been exhausted and thus once they switch to $u=a$ they need to use up all remaining inhibitors.

It is just a consequence of the continuous dependence of a solution to an ordinary differential equation on initial data and parameters that the endpoint $p_{\varepsilon}(T(\varepsilon))$ and thus the value $v(\varepsilon)$ depend continuously on $\varepsilon$. Hence, if $\hat{\varepsilon}$ is a parameter value where $v(\varepsilon)$ attains its minimum over $[0, \theta]$, then $\Gamma_{\hat{\varepsilon}}(\cdot)$ is the optimal trajectory starting at $\tilde{z}$ with correspondingly defined optimal control.

The family $\Gamma_{\varepsilon}(s), 0 \leq s \leq T(\varepsilon)$, is illustrated in Fig. 8.1 for the initial conditions $\tilde{p}=10,000$ and $\tilde{q}=3,500$. The reference trajectory is shown in blue and some sample trajectories of $\Gamma_{\varepsilon}$ are shown in black. The red curve is the curve of points when all inhibitors are being exhausted. Here the optimal control is of the type $0 s 0$ and given for $\varepsilon=\theta$, i.e. the optimal trajectory follows $u=0$ until the singular curve $\mathcal{S}$ is reached, then follows the singular arc until all inhibitors have been exhausted and finally uses $u=0$ to reach the diagonal. This is always the case for initial conditions whose available inhibitors are too small to reach region $I I$ inside the loop $\mathcal{S}$ using the control $u=a$. This is the case for this example and in fact the only extremal corresponding to this initial condition is the optimal trajectory $\Gamma_{\theta}$. On the other hand, if initial conditions have an abundance of inhibitors so that the singular arc would saturate, then optimal trajectories exit the singular arc. 


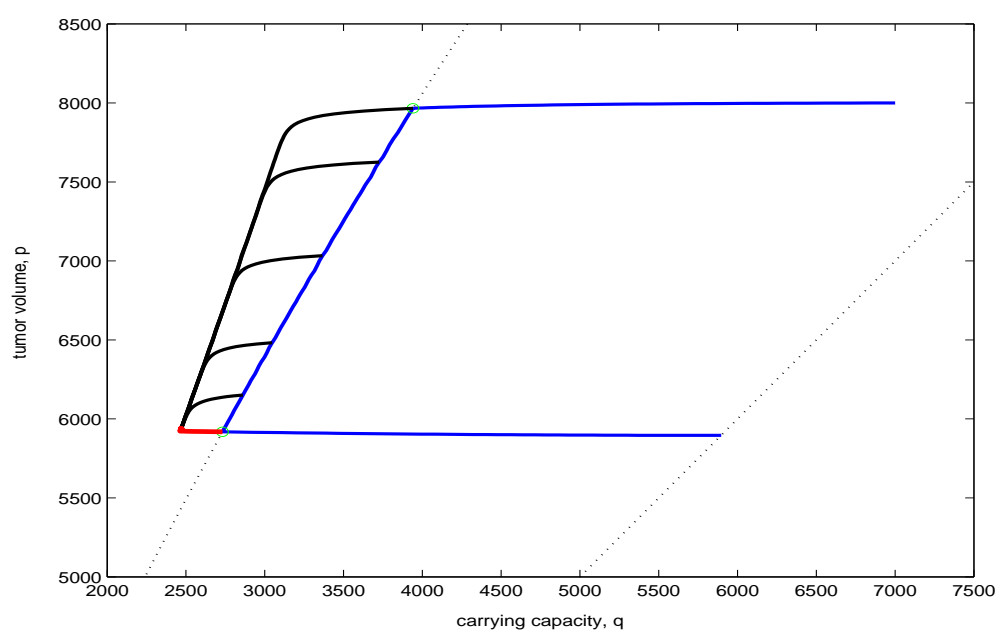

FIG. 8.2. Parameterized extremals for an initial condition in region II without saturation

(2) Let $G=I I \cup \mathcal{D}_{0} \cup\left(I I I \cap \mathcal{N}_{+}\right)$, i.e the region inside the loop $\mathcal{S}$, but 'above' the curve $\mathcal{N}_{0}$, and consider a well-posed initial condition $\tilde{z}=(\tilde{p}, \tilde{q}, 0) \in G$. Then the optimal control is initially given by $u=a$. (If the control starts with $u=0$, then the entire forward orbit lies in $G$ and in $G$ switchings from 0 to $a$ are not optimal (c.f. Propositions 7.1 and 7.2). If the $a$-trajectory starting at $\tilde{z}$ does not intersect the admissible singular arc, the optimal control is simply $u=a$ until all inhibitors have been exhausted and then $u=0$ until the diagonal is reached. Nothing else needs to done in this case. Otherwise let $\varsigma_{-}$denote the reference trajectory that starts at $\tilde{z}=(\tilde{p}, \tilde{q}, 0)$, uses the control $u=a$ until the singular arc is reached at some time $\tau$ and then, as above, follows the singular arc for time $\sigma$ until either all available inhibitors have been exhausted or the saturation point is reached. Again we use the time along the singular arc of the reference trajectory as parameter and construct the family $\Gamma_{\varepsilon}$ over the compact parameter interval $[0, \theta]$ with $\theta=\sigma$ as follows: the trajectory $\Gamma_{\varepsilon}(\cdot)$ agrees with the reference trajectory $\varsigma_{-}$along the initial segment for $u=a$ until the singular arc is reached at time $\tau$, then $\Gamma_{\varepsilon}(\cdot)$ still follows the singular arc for time $\varepsilon$ when it again switches to the control $u=a$. The end is as before: the control $u=a$ is used until all remaining inhibitors have been exhausted at which time a final segment with $u=0$ is added until the trajectory terminates at time $T$ on the diagonal. Note that $\varepsilon=0$ corresponds to the special case when the trajectory does not follow the singular arc, but continues straight with $u=a$. As above, this family contains all possible extremals that start at $\tilde{z}$, but also may have some members that are not extremals (for example, the second arc with $u=0$ may violate Proposition 7.4). The optimal trajectory is given by $\Gamma_{\hat{\varepsilon}}(\cdot)$ where $\hat{\varepsilon}$ is a minimizer of $v(\varepsilon)$ over $[0, \theta]$. In particular, in this case optimal controls at most have the form asa0 with possibly some of the pieces absent.

Fig. 8.2 shows an example of this family $\Gamma_{\varepsilon}(s), 0 \leq s \leq T(\varepsilon)$, for initial conditions $p_{0}=8,000$ and $q_{0}=7,000$. Like in Fig. 8.1 the reference trajectory $\varsigma_{-}$is shown in blue, sample trajectories from the family in black and the curve of points when all inhibitors have been exhausted is shown in red. As above, no saturation occurs and it is optimal to follow the singular arc until all inhibitors have been exhausted (the trajectory corresponding to the rightmost parameter value $\theta$ gives the minimum 
value). Also for this example this is in fact the only extremal.

This, however is no longer true when there are still inhibitors available at the saturation point. An example of this scenario is given for the initial condition $p_{0}=5,000$ and $q_{0}=2,500$ in region $I I I$. In this case, however, the numerical differences between the values of the objectives are minute. In fact, no difference in the trajectories of the family is discernable and the cost only varies between 3761.65 and 3761.98 . It is clear that, although present mathematically, these differences are of no significance and for all practical purposes one may simply continue the singular arc at saturation with $u=a$ without any noticeable loss.

It is furthermore clear that the case of initial conditions with $(\tilde{p}, \tilde{q})$ on the admissible portion of the singular arc can be analyzed in exactly the same way by setting $\tau=0$. If the initial condition lies on the inadmissible portion, optimal controls are of the form $a 0$ with all inhibitors being exhausted along $u=a$.

(3) The last case corresponds to initial conditions $(\tilde{p}, \tilde{q})$ in the region $F=(I I I \cap$ $\left.\mathcal{N}_{-}\right) \cup\left(\mathcal{S} \cap \mathcal{D}_{-}\right) \cup I V$, i.e. points that lie in $\mathcal{D}_{-}$'below' $\mathcal{N}_{0}$. These initial conditions all have relatively very large $q$-values in contrast to small $p$-values. In these cases in principle the control can start with $u=0$ and switch to $u=a$ while still in $F$. Once the control is $u=a$ the trajectory enters the region $G$ and the construction of (2) applies. Thus, and if a large amount of inhibitors is available, here the full structure 0asa0 can arise. Since trajectories eventually enter the region $G$, and this leads to a repetition of the construction, we skip a precise description of what now would be a 2-parameter family of extremals over a compact rectangle.

9. Conclusion. We presented a complete solution for a mathematical model for tumor anti-angiogenesis for the problem of optimally scheduling a given amount of inhibitors in order to minimize the primary tumor volume. Based on our theoretical analysis of the problem, for any specific initial condition the optimal solution can easily be computed numerically and as such provides a benchmark value to which other strategies should be compared. From a practical point of view, it is not realistic to employ the singular control. It is a feedback control and the required information certainly is not available, although it could be predetermined off-line from the initial condition. Naturally, strategies of the type $a 0$ which give all available inhibitors in one session are the easiest to implement in practice. It follows from our analysis that for some initial conditions these are indeed the optimal ones. This is certainly the case for initial conditions for which $a$-trajectories do not meet the admissible singular arc, but also for initial conditions when this intersection point is close to the saturation point. Indeed, the dynamics for $u \equiv a$ very much has a differential-algebraic structure with the $q$-dynamics fast and the $p$-dynamics slow. As a result, after a brief transient phase in steady-state the system essentially follows the $\dot{q}$-nullcline. This nullcline is very close to the singular curve near the saturation point and thus there the differences in the objective are almost unnoticeable. For initial conditions far away from this point the singular arc and the $\dot{q}$-nullcline are separated and then the singular control is noticeably better. Of course, only knowing the optimal solution allows to make such an analysis. However, this, and also comparisons with other models, will only be pursued elsewhere.

Acknowledgement. We would like to thank two anonymous referees for their careful reading of the paper and valuable suggestions regarding the exposition of the material. Especially thanks are due to a referee who clarifed some of the underlying medical aspects and whose comments we incorporated into section 2 . 


\section{REFERENCES}

[1] Z. Agur, L. Arakelyan, P. Daugulis and Y. Ginosar, Hopf point analysis for angiogenesis models, Discrete and Continuous Dynamical Systems, Series B, 4, No. 1, (2004), pp. 29-38

[2] B. Bonnard and J. de Morant, Toward a geometric theory in the time-minimal control of chemical batch reactors, SIAM J. Control Optim., 33, (1995), pp. 1279-1311

[3] A.E. Bryson and Y.C. Ho, Applied Optimal Control, Hemisphere Publishing, 1975

[4] L. Cesari, Optimization - Theory and Applications, Springer Verlag, New York, 1983

[5] A. d'Onofrio and A. Gandolfi, Tumour eradication by antiangiogenic therapy: analysis and extensions of the model by Hahnfeldt et al. (1999), Mathematical Biosciences, 191, (2004), pp. $159-184$

[6] A. Ergun, K. Camphausen and L.M. Wein, Optimal scheduling of radiotherapy and angiogenic inhibitors, Bulletin of Mathematical Biology, 65, (2003), pp. 407-424

[7] U. Forys, Y. Keifetz and Y. Kogan, Critical-point analysis for three-variable cancer angiogenesis models, Mathematical Biosciences and Engineering, 2, no. 3, (2005), pp. 511-525

[8] J.H. Goldie, Drug resistance in cancer: a perspective, Cancer and Metastasis Review, 20, (2001), pp. 63-68

[9] P. Hahnfeldt, D. Panigrahy, J. Folkman and L. Hlatky, Tumor development under angiogenic signaling: a dynamical theory of tumor growth, treatment response, and postvascular dormancy, Cancer Research, 59, (1999), pp. 4770-4775

[10] R.S. Kerbel, A cancer therapy resistant to resistance, Nature, 390, (1997), pp. 335-336

[11] A. Krener, The high-order maximal principle and its application to singular controls, SIAM J. Control and Optimization, 15, (1977), pp. 256-293

[12] U. Ledzewicz and H. Schättler, A synthesis of optimal controls for a model of tumor growth under angiogenic inhibitors, Proceedings of the 44th IEEE Conference on Decision and Control, Sevilla, Spain, December 2005, pp. 934-939

[13] U. Ledzewicz and H. Schättler, Optimal control for a system modelling tumor anti-angiogenesis, ICGST-ACSE Journal, 6, (2006), pp. 33-39

[14] U. Ledzewicz and H. Schättler, Application of optimal control to a system describing tumor antiangiogenesis, Proceedings of the 17th International Symposium on Mathematical Theory of Networks and Systems (MTNS), Kyoto, Japan, July 2006, pp. 478-484

[15] U. Ledzewicz and H. Schättler, Analysis of optimal controls for a mathematical model of tumor anti-angiogenesis, Optimal Control, Methods and Applications, to appear

[16] L.A. Loeb, A mutator phenotype in cancer, Cancer Research, 61, (2001), pp. 3230-3239

[17] H. Schättler and M. Jankovic, A Synthesis of time-optimal controls in the presence of saturated singular arcs, Forum Mathematicum, 5, 1993, pp. 203-241

[18] L.S. Pontryagin, V.G. Boltyanskii, R.V. Gamkrelidze and E.F. Mishchenko, The Mathematical Theory of Optimal Processes, MacMillan, New York, (1964) 